\title{
Benzotriazole-mediated amidoalkylations of nitroalkanes, nitriles, alkynes and esters
}

\author{
Alan R. Katritzky, ${ }^{a^{*}}$ Ashraf A. A. Abdel-Fattah ${ }^{\text {a,b }}$, and Ilhami Celik ${ }^{\text {a,c }}$ \\ ${ }^{a}$ Center for Heterocyclic Compounds, Department of Chemistry, University of Florida, \\ Gainesville, FL 32611-7200 \\ ${ }^{b}$ Department of Chemistry, Faculty of Science, Benha University, Benha, Egypt \\ ${ }^{c}$ Department of Chemistry, Faculty of Science, Anadolu University, 26470 Eskisehir, Turkey \\ E-mail: Katritzky@chem.ufl.edu
}

\begin{abstract}
Reactions of readily available and stable $N$-( $\alpha$-amidoalkyl)benzotriazoles 1 (derived from a variety of aliphatic, aromatic or heterocyclic aldehydes) with diverse nitroalkanes, nitriles, alkynes and esters afforded $N$-( $\beta$-nitroalkyl)amides 4 (54-96\%), $N$ - $(\beta$-cyanoalkyl)amides 6 (58-88\%), $N$-acylpropargylamines $11(41-87 \%)$ and esters of $\beta$ - $N$-acylamino acids 13 (68-96\%), respectively.
\end{abstract}

Keywords: Amidoalkylation, $N$-( $\beta$-nitroalkyl)amides, $N$ - $(\beta$-cyanoalkyl)-amides, $N$-acylpropargylamines, $\beta$ - $N$-acylamino acids

\section{Introduction}

Amidoalkylations have attracted attention in organic synthesis as a valuable alternative to the Mannich reaction ${ }^{1}$ since they provide ready access to a wide variety of $\alpha$-substituted amines. ${ }^{2}$ Amidoalkylation of $\pi$-nucleophiles, including alkenes, allenes, alkynes and (hetero)aromatic systems, is a common step in the synthesis of nitrogen heterocycles, ${ }^{2 a, b}$ alkaloids, ${ }^{3}$ and other nitrogen-containing biologically active compounds. ${ }^{2 b}$ Intramolecular amidoalkylations have been applied to the synthesis of monocyclic, ${ }^{4}$ bicyclic and polycyclic systems with stereocontrol. ${ }^{5}$

Benzotriazole mediated amidoalkylations introduced in $1988,{ }^{6}$ offer advantages over previously reported methods as was already demonstrated for the amidoalkylation of (i) Grignards, ${ }^{6}$ (ii) malonates and acetoacetates, ${ }^{7}$ (iii) cyanide anion, ${ }^{8}$ (iv) mercaptans and alcohols, ${ }^{9}$ (v) electron-rich (hetero)aromatics, ${ }^{10}$ and (vi) amines ${ }^{11}$ (Scheme 1).

\footnotetext{
* Alan Katritzky was Chairman of the RSC Heterocyclic Group during the period 1967-1969.
} 
We now report that $N$-( $\alpha$-amido-alkyl)benzotriazoles are also advantageous for the amidoalkylation of nitroalkanes, nitriles, acetylenes, and esters to produce the corresponding novel $\beta$-functionalized amides.

\section{Results and Discussion}

\section{Preparation of $N$-( $\alpha$-amidoalkyl)benzotriazoles $1 \mathrm{a}-\mathbf{i}$}

Amidoalkylation reagents $\mathbf{1 a}-\mathbf{i}$ were easily prepared by the well-established condensation of benzotriazole, an aldehyde, and an amide in the presence of a catalytic amount of $p$ toluenesulfonic acid in refluxing toluene with azeotropic removal of water. ${ }^{12}$ Both aliphatic and aromatic aldehydes gave stable products $\mathbf{1}$ in good yields, which were fully characterized on the basis of their ${ }^{1} \mathrm{H}$ and ${ }^{13} \mathrm{C}$ NMR spectra and elemental analyses. The ${ }^{1} \mathrm{H}$ and ${ }^{13} \mathrm{C} \mathrm{NMR}$ spectra of these $N$-( $\alpha$-amidoalkyl)benzotriazoles confirmed that the products $\mathbf{1 a}-\mathbf{i}$ are all benzotriazol-1-yl compounds with no isomerization to benzotriazol-2-yl isomers.

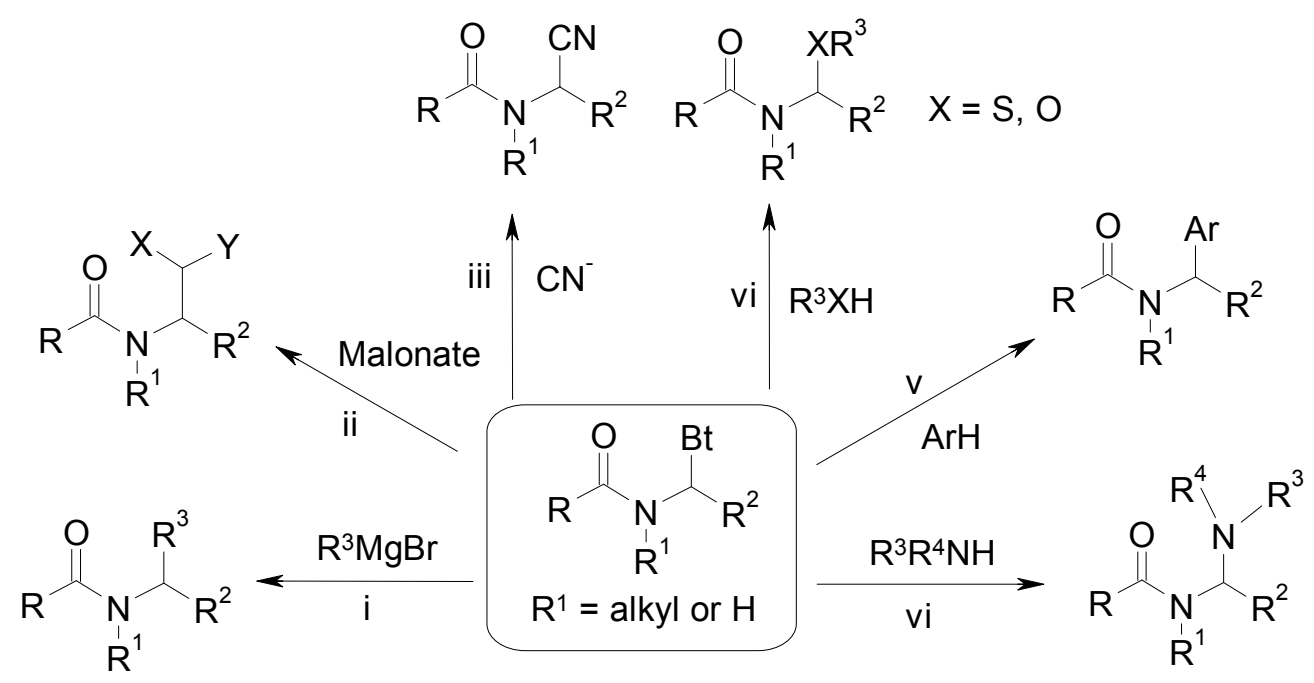

\section{Scheme 1}

\section{Synthesis of $\boldsymbol{N}$ - $(\boldsymbol{\beta}$-nitroalkyl) amides}

Nitronate salts act as carbon nucleophiles in the well-known Michael, ${ }^{13}$ Henry, ${ }^{14}$ Knoevenagel, ${ }^{15}$ and nitro-Mannich reactions, ${ }^{16}$ providing valuable derivatives in which the nitro group can be retained, reductively eliminated, or transformed to provide ketones, ${ }^{17}$ amines, ${ }^{18}$ oximes, ${ }^{19}$ or nitriles. $^{20}$ Therefore, methodologies to introduce the nitro group into a molecular framework along with other functionalities have gained momentum.

$N$ - $\left(\alpha\right.$-Amidoalkyl)benzotriazoles 1 ( $\mathrm{R}^{1}$ derived from benzaldehyde) react with sodium alkanenitronates in $\mathrm{DMF}^{21}$ Encouraged by reports that $N$ - $(\beta$-nitroalkyl)amides of type 4 represent viable intermediates to $N$-protected $\alpha$-amino acids, ${ }^{22}$ we have now generalized this efficient entry to $N$-( $\beta$-nitroalkyl)amides (Scheme 2$)$. 
Reacting 1f with 2 molar equivalents of potassium methanenitronate, generated in situ gave $N$-( $\beta$-nitroalkyl) amide 4a (79\%), we note that neither $\mathrm{NaH}$ nor $\mathrm{NaOCH}_{3}$ promote this reaction. The scope of the reaction is wide: $N$ - $(\alpha$-amidoalkyl)benzotriazoles derived from aliphatic, aromatic, and heteroaromatic aldehydes and various primary amides, were reacted with diverse nitroalkanes to provide the corresponding $\beta$-nitroalkyl amides $\mathbf{4 a - 1}$ (Scheme 2 and Table $1)$.

Formation of the nitroamides of type 4 is indicated by the loss of benzotriazole signals in ${ }^{1} \mathrm{H}$ and $\mathrm{C}^{13} \mathrm{NMR}$. In the ${ }^{13} \mathrm{C}$ NMR spectra of the compounds 4 , resonances arising from amide carbonyl and carbons adjacent to the nitro group are found in the regions of 166.3-178.2 ppm and 86.0-97.0 ppm, respectively and their structures were also confirmed by elemental analysis.

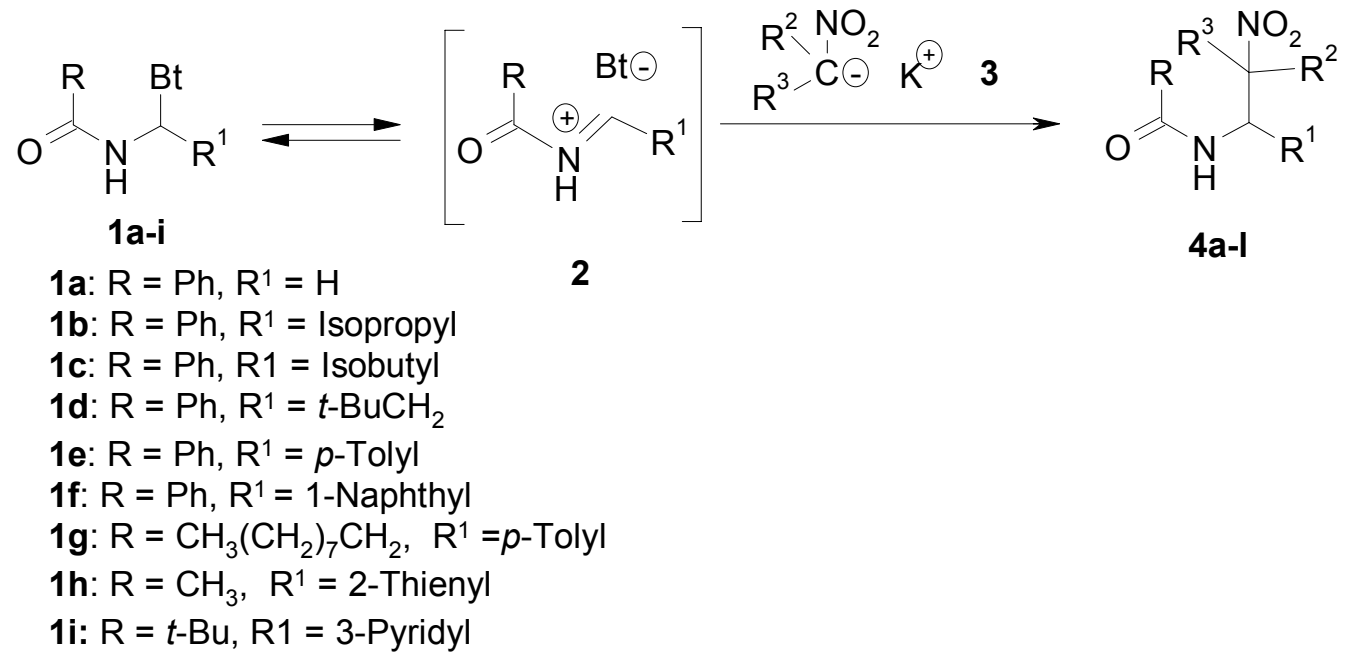

For designation of $\mathrm{R}, \mathrm{R}^{1} \mathrm{R}^{2}$ and $\mathrm{R}^{3}$ in $\mathbf{4}$ see Table 1

\section{Scheme 2}

Table 1. Preparation of $N$ - $(\beta$-nitroalkyl $)$ amides $\mathbf{4 a - l}$

\begin{tabular}{cccccc}
\hline Compd & $\mathbf{R}$ & $\mathbf{R}^{\mathbf{1}}$ & $\mathbf{R}^{\mathbf{2}}$ & $\mathbf{R}^{\mathbf{3}}$ & Yield \% \\
\hline $\mathbf{4 a}$ & $\mathrm{Ph}$ & $p$-Tolyl & $\mathrm{H}$ & $\mathrm{H}$ & 79 \\
$\mathbf{4 b}$ & $\mathrm{Ph}$ & $p$-Tolyl & $\mathrm{CH}_{3}$ & $\mathrm{CH}_{3}$ & 82 \\
$\mathbf{4 c}$ & $\mathrm{Ph}$ & Isopropyl & $\mathrm{H}$ & $\mathrm{H}$ & 85 \\
$\mathbf{4 d}$ & $\mathrm{Ph}$ & Isopropyl & $\mathrm{CH}_{3}$ & $\mathrm{CH}_{3}$ & 87 \\
$\mathbf{4 e}$ & $\mathrm{Ph}$ & Isopropyl & $\mathrm{H}$ & $\mathrm{Et}$ & 96 \\
$\mathbf{4 f}$ & $\mathrm{Ph}$ & $\mathrm{H}$ & $\mathrm{H}$ & $\mathrm{H}$ & 54 \\
$\mathbf{4 g}$ & $\mathrm{Ph}$ & $\mathrm{H}$ & $\mathrm{CH}_{3}$ & $\mathrm{CH}_{3}$ & 86 \\
$\mathbf{4 h}$ & $\mathrm{Ph}$ & Isopropyl & \multicolumn{2}{c}{$\left(\mathrm{CH}_{2}\right)_{5}$} & 93 \\
$\mathbf{4 i}$ & $\mathrm{CH}_{3}(\mathrm{CH})_{8}$ & $p$-Tolyl & $\mathrm{H}$ & $\mathrm{CH}_{3}$ & 89 \\
$\mathbf{4 j}$ & $\mathrm{Ph}$ & $t$-BuCH & $\mathrm{CH}$ & 81 \\
$\mathbf{4 k}$ & $\mathrm{CH}_{3}$ & $2-$ Thienyl & $\mathrm{H}$ & $\mathrm{Et}_{3}$ & 58
\end{tabular}


Previous approaches to $N$-( $\beta$-nitroalkyl) amides include: (i) nitroacetamidation of olefins by $\mathrm{NaNO}_{2}$-ceric ammonium nitrate-acetonitrile reagent ${ }^{23}$ (examples limited to cyclic olefins); (ii) nucleophilic substitution of $\alpha$-bromoalkyl amides with lithium alkanenitroates ${ }^{24}$ (examples limited to $\alpha$-bromoglycine derivatives); and (iii) reactions of sodium nitronate with $\alpha$-amidoalkyl sulfones ${ }^{22 \mathrm{~b}}$ (examples limited the amidoalkylation of nitromethane). The previously described protocol can tolerate variation in each component; the 12 examples of Table 1 demonstrate that $N$-( $\alpha$-amidoalkyl)benzotriazoles derived from formaldehyde, aliphatic, aromatic or heterocyclic aldehydes and diverse amides can amidoalkylate primary and secondary nitroalkanes providing the expected $\beta$-nitroalkyl amides in yields ranging from $54 \%$ to $96 \%$ (average $77 \%$ ).

\section{Synthesis of $N$ - $(\beta$-cyanoalkyl)amides}

$\beta$-Amino acids and their derivatives show a variety of interesting biological properties. ${ }^{25}$ Therefore, a new strategy for $N$-( $\beta$-cyanoalkyl)amide synthesis can provide valuable intermediates for $\beta$-amino acids. ${ }^{26}$

$N$-( $\beta$-Cyanoalkyl)amides were previously prepared (Scheme 3): (i) by 2,3-bond cleavage of azetidinones promoted by trimethylsilyl trifluoromethanesulfonate (TMSOTf) in a cyano groupcontaining solvent; ${ }^{27}$ (ii) nucleophilic ring opening of $\mathrm{N}$-acyl aziridines with trimethylsilyl cyanide (TMSCN) triggered by tetrabutylammonium fluoride; ${ }^{28}$ (iii) Lewis acid-catalyzed cyanide addition of $\alpha$-iminoalkyl amide using trimethylsilyl cyanide (TMSCN) ${ }^{26 b}$ and (iv) N-N bond cleavage of pyrazolidine derivatives having a thioketal moiety with $\mathrm{SmI}_{2} \cdot{ }^{29}$ However, none can be considered a general methodology; overall methods (i) - (iv) report a total of just nine examples. Recently, we reported reactions of metallated nitriles with $\mathrm{N}$-sulfonyl- ${ }^{30} \mathrm{~N}$-acyl-, ${ }^{31}$ and $N$-( $\alpha$-aminoalkyl)benzotriazoles ${ }^{32}$ to provide novel approaches to cyano- sulfones, ketones and amines, respectively. We now disclose a logical extension of this synthetic strategy by the utilization of $N$-( $\alpha$-amidoalkyl)benzotriazoles as electrophilic substrates for metalated nitriles.

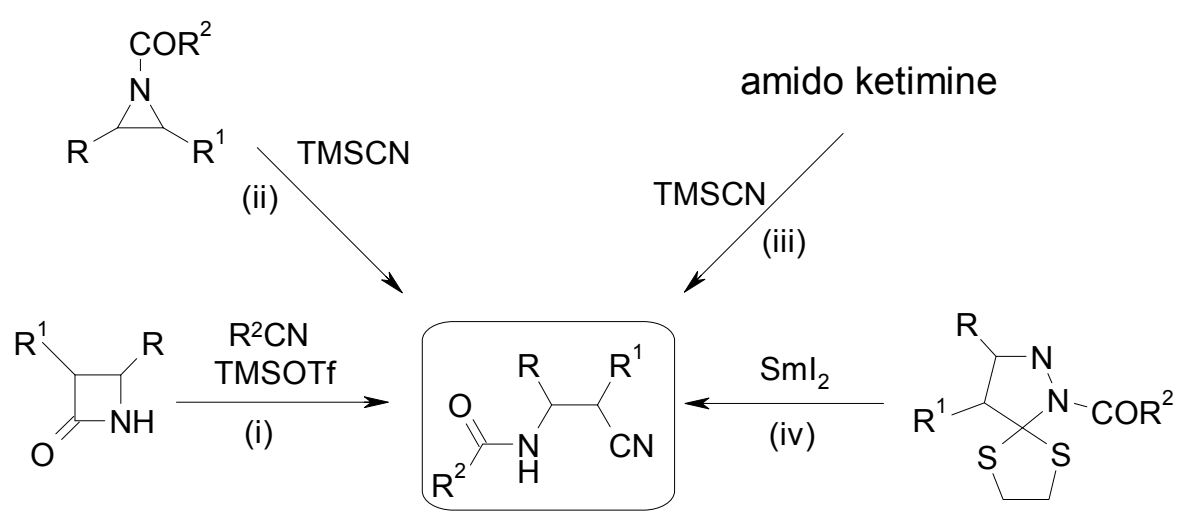

\section{Scheme 3}


Trials with different base-solvent couples disclosed that phenylacetonitrile treated with 1.2 molar equivalent of $t$ - $\mathrm{BuOK}$ in DMSO followed by subsequent addition of $\alpha$ (amidoalkyl)benzotriazole 1a solution in DMSO at $15{ }^{\circ} \mathrm{C}$ provided $58 \%$ of the amidomethylated product 6a. The significant acidity of the hydrogen linked to the nitrogen atom in $\mathbf{1}$ and anionexchange explains the bis-amide 8 byproduct.

Using these optimized conditions, potassio-derivatives of $\mathbf{5} \mathbf{a}-\mathbf{e}$ (generated from primary or secondary nitriles) reacted with diverse 1 to give 10 examples of the expected monoamidoalkyation products 6 in yields $61 \%$ to $92 \%$ (Scheme 4 and Table 2). For $\beta$-amidoalkyl nitriles 6 containing two asymmetric carbon atoms, the reaction shows little stereoselectivity; in nearly all cases two stereoisomers were isolated in about 1:1 ratio. Assignment of the two stereoisomers as syn and anti was accomplished by utilizing the reasoning of Carlier et al on $\beta$ hydroxy nitriles, ${ }^{33}$ and our own recent results with $\beta$-aminoalkyl nitriles. ${ }^{32}$ The reaction of $\mathbf{1 h}$ with $\mathbf{5 c}$ under the same conditions provided the doubly amidoalkylated product 7 (46\%) (Scheme 4, Table 2).

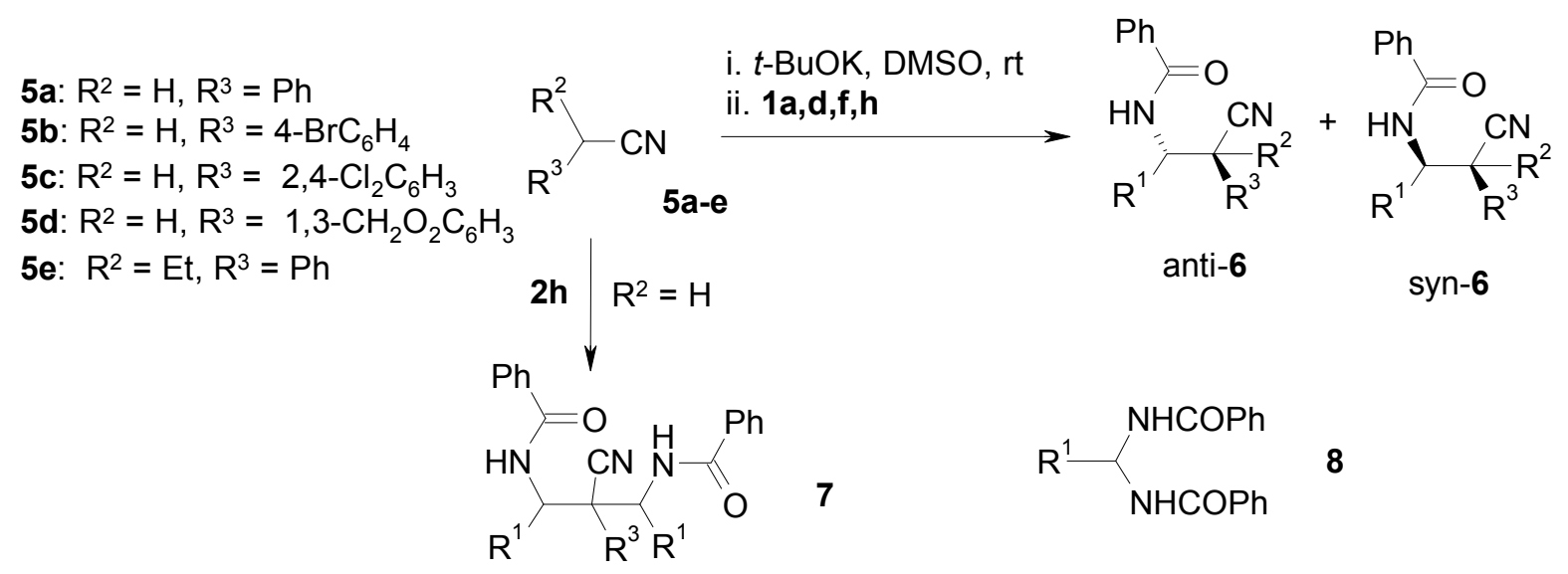

For designation of $\mathrm{R}^{1} \mathrm{R}^{2}$ and $\mathrm{R}^{3}$ in $\mathbf{6}$ and 7 see Table 2.

\section{Scheme 4}

Table 2. Synthesis of $\beta$-cyanoalkyl amides 6 and 7

\begin{tabular}{ccccccc}
\hline Compd & $\mathbf{R}^{\mathbf{1}}$ & $\mathbf{R}^{\mathbf{2}}$ & $\mathbf{R}^{\mathbf{3}}$ & $\begin{array}{c}\text { Yield \% of } \\
\text { anti-6 }\end{array}$ & $\begin{array}{c}\text { Yield \% of } \\
\text { syn-6 }\end{array}$ & $\begin{array}{c}\text { Overall } \\
\text { yield \% }\end{array}$ \\
\hline $\mathbf{6 a}$ & $t-\mathrm{BuCH}_{2}$ & $\mathrm{H}$ & $\mathrm{Ph}$ & 78 & - & 78 \\
$\mathbf{6 b}$ & $t-\mathrm{BuCH}_{2}$ & $\mathrm{H}$ & $4-\mathrm{BrC}_{6} \mathrm{H}_{4}$ & 87 & - & 87 \\
$\mathbf{6 c}$ & $t-\mathrm{BuCH}_{2}$ & $\mathrm{H}$ & $\mathrm{Ben}^{\mathrm{a}}$ & 45 & 41 & 86 \\
$\mathbf{6 d}$ & $t-\mathrm{BuCH}_{2}$ & $\mathrm{Et}$ & $\mathrm{Ph}$ & 45 & 43 & 88 \\
$\mathbf{6 e}$ & $1-\mathrm{Naphthyl}$ & $\mathrm{Et}$ & $\mathrm{Ph}$ & 41 & 32 & 73 \\
$\mathbf{6 f}$ & $\mathrm{H}$ & $\mathrm{H}$ & $\mathrm{Ph}$ & - & - & 58 \\
$\mathbf{6 g}$ & $\mathrm{H}$ & $\mathrm{H}$ & $2,4-\mathrm{Cl}_{2} \mathrm{C}_{6} \mathrm{H}_{3}$ & - & - & 65
\end{tabular}




\begin{tabular}{ccccccc}
$\mathbf{6 h}$ & $\mathrm{H}$ & $\mathrm{Et}$ & $\mathrm{Ph}$ & - & - & 61 \\
$\mathbf{7}$ & 1-Naphthyl & - & $2,4-\mathrm{Cl}_{2} \mathrm{C}_{6} \mathrm{H}_{3}$ & & - & 44 \\
\hline
\end{tabular}

\section{${ }^{\mathrm{a}} 1,3$-Benzo[1,3]dioxol-4-yl}

Novel structures of $\mathbf{6}$ and $\mathbf{7}$ were supported by their elemental analyses and spectral data. The ${ }^{13} \mathrm{C}$ NMR spectra of the amides show the absorption peak for the carbonyl group in the region of 168.4-166.8 ppm and for the carbon directly attached to the amide nitrogen at $56.1-53.1 \mathrm{ppm}$.

\section{Synthesis of $N$-acylpropargylamines}

Propargylamines are important therapeutic agents: ${ }^{34}$ inhibitors of monoamine oxidase B and aldehyde dehydrogenase enzymes ${ }^{35}$ and potential antifungal agents. ${ }^{36}$ They are also precursors for allylic amines and other targets. ${ }^{37}$ Propargylamines were previously prepared by: (i) metalcatalyzed three-component coupling of aldehyde, alkyne, and secondary amine; ${ }^{38}$ (ii) metalcatalyzed addition of alkynes to enamines; ${ }^{39}$ (iii) $\mathrm{TiCl}_{4}$-mediated amination of propargylic esters; ${ }^{40}$ (iv) transition metal promoted addition reaction of terminal alkynes to imines; ${ }^{41}$ and (v) alkynylation of $N$-( $\alpha$-aminoalkyl)benzotriazoles with lithium alkynides ${ }^{42}$ or dialkynyldiethylaluminates ${ }^{43}$ (Scheme 5). We now report the synthesis of $N$-acyl- $\alpha$-propargylamines 11 (Scheme 6), which can serve as precursors for the corresponding $\alpha$-propargylamines. ${ }^{40}$

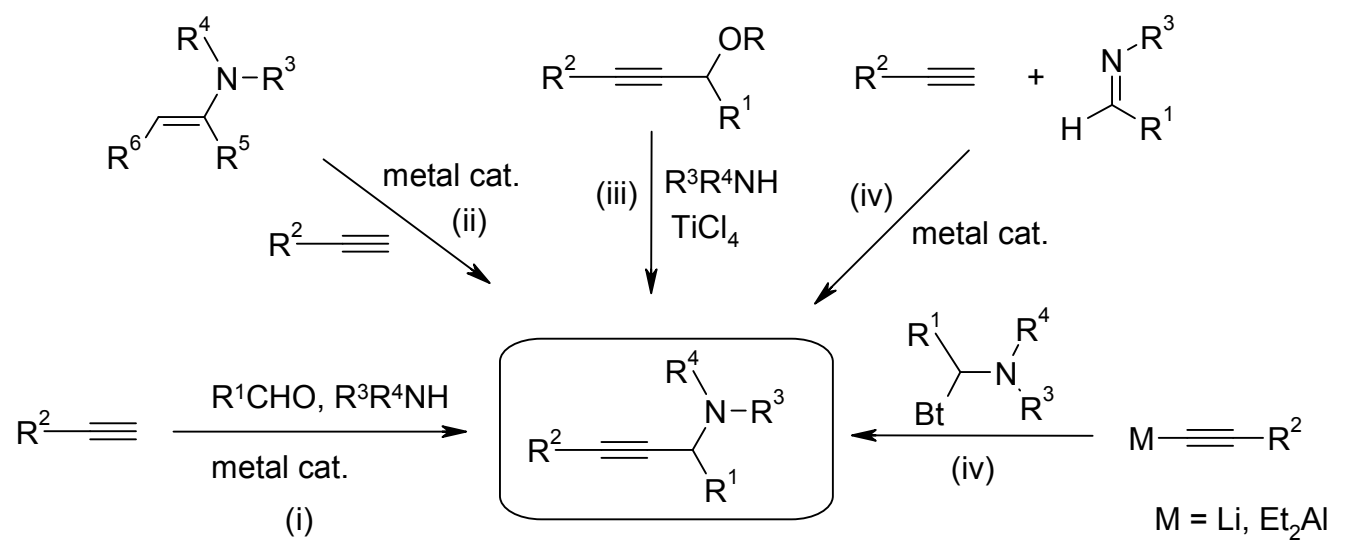

\section{Scheme 5}

2-Phenylacetylene 9a was treated with 1.2 equiv. of $n$-BuLi to give 10a in situ which on treatment with $1 \mathbf{c}, \mathbf{d}$ at $-78{ }^{\circ} \mathrm{C}$ in THF afforded $53 \%$ and $63 \%$ of 11 a and $11 \mathbf{b}$, respectively. The molar equiv. of lithium alkynide could be replaced by 1.9 equiv. of alkynylmagnesium bromide 10 (prepared by treating 9 with 1.65 equiv of $\mathrm{EtMgBr}$ ) at rt affording the corresponding $\mathrm{N}$ acylpropargylic amides 11c-g in 47-87\% yields (Scheme 6 and Table 3). Structures 11c-g were characterized by NMR spectroscopy. The ${ }^{1} \mathrm{H}$ NMR of $\mathbf{1 1}$ showed the characteristic peak of NH 
as doublet in the region 6.20-6.41 ppm and the peaks of the two acetylenic carbons appeared in the ${ }^{13} \mathrm{C}$ NMR spectra in the regions $83.7-88.7 \mathrm{ppm}$ and $76.4-84.3 \mathrm{ppm}$.

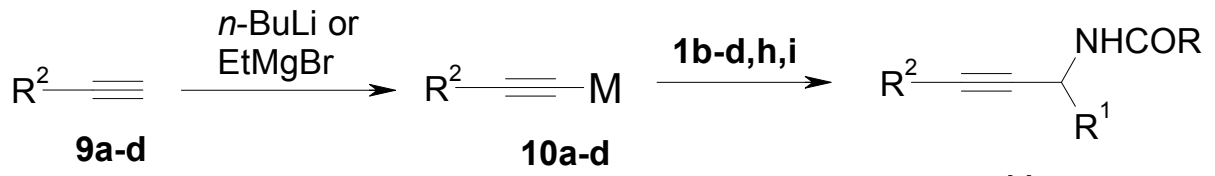

9a: $\mathrm{R}^{2}=\mathrm{Ph} ; \mathbf{9 b}: \mathrm{R}^{2}=p$-Tolyl

11a-g

9c: $\mathrm{R}^{2}=n$-Pentyl; 9d: $\mathrm{R}^{2}=n$-Hexyl

For designation of $\mathrm{R}, \mathrm{R}^{1}$ and $\mathrm{R}^{2}$ in $\mathbf{1 1}$ see Table 3.

\section{Scheme 6}

\section{Synthesis of esters of $\boldsymbol{\beta}$ - $\boldsymbol{N}$-acylamino acids 13}

$\beta$-Amino carbonyl compounds are precursors for biologically important $\beta$-lactams and $\beta$-amino acids. ${ }^{44}$ Therefore, a number of synthetic pathways have been devised for their preparation including (Scheme 7), (i) enantioselective hydrogenations of $\beta$-amine acrylate; ${ }^{45}$ (ii) asymmetric additions of different enolate equivalents to imines, ${ }^{46}$ and (iii) reactions of $N-(\alpha-$ aminoalkyl)benzotriazoles with ketene silyl acetals catalyzed by either lanthanide triflate ${ }^{47}$ or methyl lithium. ${ }^{48}$ We now report that reaction of esters with 1 provides a novel access to $\beta$ - $(N-$ protected-amino)alkyl esters 13 (Scheme 8).

Table 3. Synthesis of $N$-acyl propargylic amines 11

\begin{tabular}{cccccc}
\hline Compd & $\mathrm{R}$ & $\mathrm{R}^{1}$ & $\mathrm{R}^{2}$ & $\mathrm{M}$ & Yield \% \\
\hline 11a & $\mathrm{Ph}$ & $t$-BuCH & $\mathrm{Ph}$ & $\mathrm{Li}$ & 53 \\
11b & $\mathrm{Ph}$ & Isobutyl & $\mathrm{Ph}$ & $\mathrm{Li}$ & 63 \\
11c & 4-Tolyl & Isobutyl & $\mathrm{Ph}$ & $\mathrm{MgBr}$ & 86 \\
11d & 4-Tolyl & Isopropyl & $\mathrm{Ph}$ & $\mathrm{MgBr}$ & 85 \\
11e & $n$-Pentyl & Isobutyl & $\mathrm{Ph}$ & $\mathrm{MgBr}$ & 80 \\
11f & n-Hexyl & 2-Thienyl & $\mathrm{CH}_{3}$ & $\mathrm{MgBr}$ & 41 \\
11g & $\mathrm{Ph}$ & 3-Pyridyl & $t$ - $\mathrm{Bu}$ & $\mathrm{MgBr}$ & 87 \\
\hline
\end{tabular}




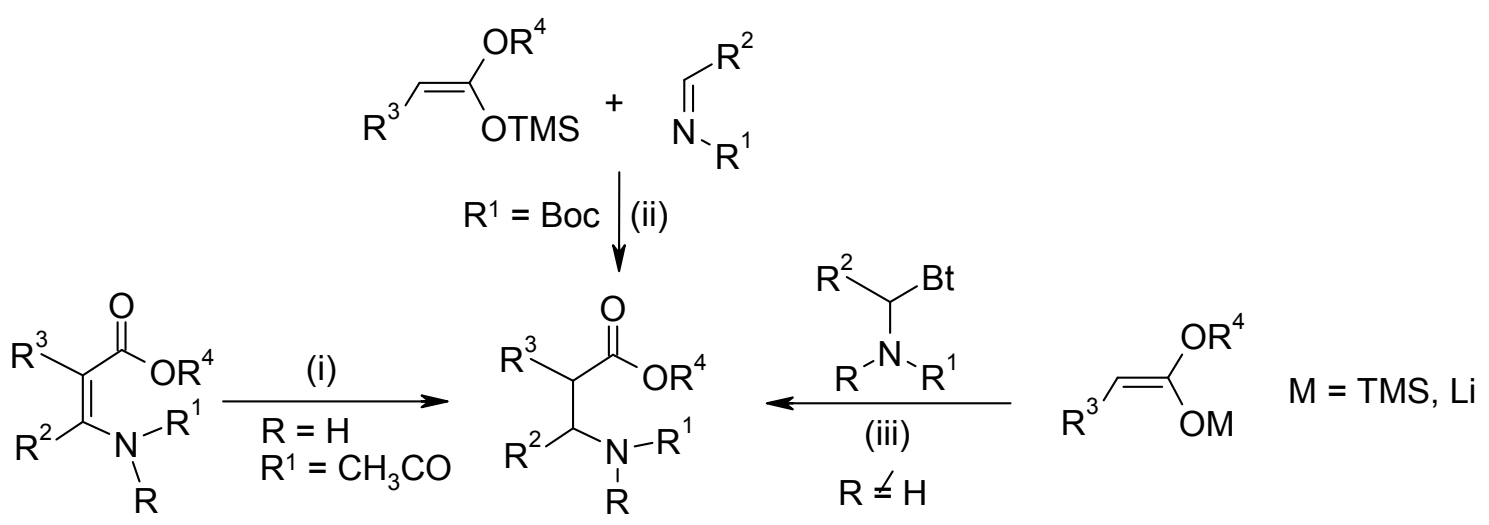

\section{Scheme 7}

Reactions of lithium or potassium enolates, prepared in situ by treating the corresponding ester itself 12a-c with LDA in THF or $t$-BuOK in DMSO, with $\mathbf{1 b}-\mathbf{d}$ provided $\beta$-( $N$-protectedamino)alkyl esters 13a-e in moderate to excellent yields (Scheme 8 and Table 4).

Previously malonates and acetoacetates ${ }^{7}$ were reacted with 1 in the presence of anhydrous aluminum chloride and gave the amidoalkyated products of type $\mathbf{1 3}$ in moderate yields. The efficiency of the nucleophilic substitution of benzotriazole from compounds of type $\mathbf{1}$ is demonstrated by comparison of the electrophilic conditions. Ethyl benzoylacetate (12d), methyl and benzyl acetoacetate (12e,f), and ethyl malonate $(\mathbf{1 2 g})$, each reacted efficiently with $\mathbf{1}$ in the presence of 1.2 equiv. $t$-BuOK in DMSO at $\mathrm{rt}$, to give the amidoalkylated derivatives $\mathbf{1 3 f}-\mathbf{h}$ (84-96\%) (Scheme 7 and Table 4). The ${ }^{1} \mathrm{H}$ NMR spectra of 13 showed that while 13c and 13d were diastereomeric mixtures $13 \mathbf{a}, \mathbf{b}$ and $13 \mathbf{e}-\mathbf{g}$ were single diastereomers. In the ${ }^{13} \mathrm{C} N M R$ spectra of $\mathbf{1 3}$ the characteristic signals of ester and amide carbonyls appeared at 172.6-173.2 ppm and 167.2-169.2 ppm.
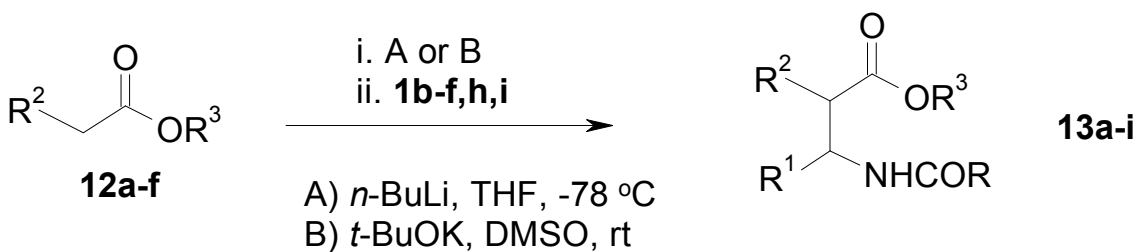

For designation of $\mathrm{R}, \mathrm{R}^{1}, \mathrm{R}^{2}$ and $\mathrm{R}^{3}$ in $\mathbf{1 3}$ see Table 4 .

\section{Scheme 8}

Table 4. Synthesis of esters of $\beta$ - $N$-acylamino acids $\mathbf{1 3}$

\begin{tabular}{lcccccc}
\hline Compd & $\mathrm{R}$ & $\mathrm{R}^{1}$ & $\mathrm{R}^{2}$ & $\mathrm{R}^{3}$ & Method & Yield \% \\
\hline 13a & $\mathrm{Ph}$ & $t$ - $\mathrm{BuCH}_{2}$ & $\mathrm{Ph}$ & $\mathrm{Me}$ & $\mathrm{A}$ & 68 \\
13b & $\mathrm{Ph}$ & Isobutyl & 1-Naphthyl & $\mathrm{Me}$ & $\mathrm{B}$ & 92 \\
13c & $\mathrm{Ph}$ & Isopropyl & 1-Naphthyl & $\mathrm{Me}$ & $\mathrm{B}$ & 89
\end{tabular}




\begin{tabular}{lcccccc} 
13d & $\mathrm{Me}$ & 2 -Thienyl & $\mathrm{Ph}$ & $\mathrm{Me}$ & $\mathrm{B}$ & 75 \\
13e & $\mathrm{Ph}$ & $t$-BuCH $\mathrm{Bu}_{2}$ & $\mathrm{PhCO}$ & $\mathrm{Et}$ & $\mathrm{B}$ & 84 \\
13f & $\mathrm{Ph}$ & $p$-Tolyl & $\mathrm{CH}_{3} \mathrm{CO}$ & $\mathrm{Me}$ & $\mathrm{B}$ & 88 \\
13g & $\mathrm{Ph}$ & 1 -Naphthyl & $\mathrm{CH}_{3} \mathrm{CO}$ & $\mathrm{Bn}$ & $\mathrm{B}$ & 96 \\
\hline
\end{tabular}

In summary, we have developed convenient approaches for $N$ - $(\beta$-nitroalkyl $)$-amides, $N-(\beta$ cyanoalkyl) amides, $N$-acylpropargylamines and esters of $\beta$-N-acylamino acids by the amidoalkylation of nitroalkanes, nitriles, alkynes, and esters and $\beta$-keto esters with $N-(\alpha-$ amidoalkyl)benzotriazoles. The adopted procedures are simple and applicable to the preparation of amidoalkylation products derived from formaldehyde, aliphatic, and (hetero) aromatic aldehydes. In all cases, the $N$-protected amine derivatives were produced in high yields that make the use of $N$-( $\alpha$-amidoalkyl)benzotriazoles as $N$-acyliminium ion equivalents advantageous.

\section{Experimental Section}

General Procedures. All reactions were carried out under an atmosphere of nitrogen, unless otherwise specified. Glassware was routinely oven-dried at $160{ }^{\circ} \mathrm{C}$ for a minimum of $4 \mathrm{~h}$ and then connected to a vacuum line before assembling under a dry argon stream. Column chromatography was performed on silica gel 200-425 mesh. THF was distilled from sodiumbenzophenone ketyl and DMSO was dried over molecular sieves prior to use. $N$-( $\alpha$-Amidoalkyl) benzotriazoles 1 were prepared according to literature procedures. ${ }^{12}$

General procedure for the preparation of $\boldsymbol{N}$-( $\boldsymbol{\beta}$-nitroalkyl) amides 4a-l. A mixture of nitroalkane ( $4 \mathrm{mmol})$ and potassium $t$-butoxide $(0.45 \mathrm{~g}, 4 \mathrm{mmol})$ in DMSO $(10 \mathrm{~mL})$ was stirred at room temperature for $40 \mathrm{~min}$. To the resulting solution $N-(\alpha-$ amidoalkyl)benzotriazoles $1(2 \mathrm{mmol})$ in DMSO $(10 \mathrm{~mL})$ was added dropwise and the mixture was stirred at room temperature for $8 \mathrm{hrs}$. The mixture was poured into water $(40 \mathrm{~mL})$, acidified with acetic acid, and then extracted with ethyl acetate $(3 \times 30 \mathrm{~mL})$. The extracts were washed with water, dried over $\mathrm{MgSO}_{4}$ and the solvent removed under reduced pressure. The residue was placed in a silica-gel column and eluted with hexanes/ EtOAc 5:1 to give 4.

$\mathrm{N}$-(2-Nitro-1-p-tolylethyl)benzamide (4a). Colorless microcrystals (79\%), mp $147-148{ }^{\circ} \mathrm{C} .{ }^{1} \mathrm{H}$ NMR $\delta 7.77(\mathrm{~d}, J=7.6 \mathrm{~Hz}, 2 \mathrm{H}), 7.53-7.38(\mathrm{~m}, 3 \mathrm{H}), 7.24(\mathrm{~d}, J=7.8 \mathrm{~Hz}, 2 \mathrm{H}), 7.23-7.15(\mathrm{~m}$, $3 \mathrm{H}), 5.83$ (q, $J=6.7 \mathrm{~Hz}, 1 \mathrm{H}), 4.99$ (dd, $J=12.9,6.6 \mathrm{~Hz}, 1 \mathrm{H}), 4.78$ (dd, $J=12.9,6.6 \mathrm{~Hz}, 1 \mathrm{H})$, 2.33 (s, 3H). ${ }^{13} \mathrm{C}$ NMR $\delta$ 167.1, 138.7, 133.4, 133.3, 132.0, 129.9, 128.6, 127.0, 126.3, 78.2, 51.4, 21.1. Anal. Calcd. For $\mathrm{C}_{16} \mathrm{H}_{16} \mathrm{~N}_{2} \mathrm{O}_{3}$ : C, 67.59; H, 5.67; N, 9.85. Found: C, 67.97; H, 5.72; $\mathrm{N}, 9.77$.

$\mathrm{N}$-(2-Methyl-2-nitro-1-p-tolylpropyl)benzamide (4b). Colorless plates (82\%), mp 162-163 ${ }^{\circ} \mathrm{C}$. ${ }^{1} \mathrm{H}$ NMR $\delta 7.83(\mathrm{~d}, J=7.0 \mathrm{~Hz}, 2 \mathrm{H}), 7.70(\mathrm{~d}, J=8.9 \mathrm{~Hz}, 1 \mathrm{H}), 7.53-7.43(\mathrm{~m}, 3 \mathrm{H}), 7.12(\mathrm{~s}, 4 \mathrm{H})$, $5.48(\mathrm{~d}, J=9.7 \mathrm{~Hz}, 1 \mathrm{H}), 2.31(\mathrm{~s}, 3 \mathrm{H}), 1.79(\mathrm{~s}, 3 \mathrm{H}), 1.57$ (s, 3H). ${ }^{13} \mathrm{C}$ NMR $\delta 166.5,138.5,133.8$, 
133.1, 131.9, 129.4, 128.7, 127.5, 127.0, 90.5, 59.1, 25.8, 23.5, 21.0. Anal. Calcd. For $\mathrm{C}_{18} \mathrm{H}_{20} \mathrm{~N}_{2} \mathrm{O}_{3}: \mathrm{C}, 69.21 ; \mathrm{H}, 6.45 ; \mathrm{N}, 8.97$. Found: $\mathrm{C}, 68.92 ; \mathrm{H}, 6.44 ; \mathrm{N}, 9.16$.

$\mathrm{N}$-(2-Methyl-1-nitromethylpropyl)benzamide (4c). Colorless plates (85\%), mp 142-143 ${ }^{\circ} \mathrm{C}$. ${ }^{1} \mathrm{H}$ NMR $\delta 7.78(\mathrm{~d}, J=7.1 \mathrm{~Hz}, 2 \mathrm{H}), 7.56-7.42(\mathrm{~m}, 3 \mathrm{H}), 6.67(\mathrm{~d}, J=8.9 \mathrm{~Hz}, 1 \mathrm{H}), 4.76(\mathrm{dd}, J=$ $12.9,5.6 \mathrm{~Hz}, 1 \mathrm{H}), 4.63(\mathrm{dd}, J=12.9,3.8 \mathrm{~Hz}, 1 \mathrm{H}), 4.46-4.37(\mathrm{~m}, 1 \mathrm{H}), 2.08-1.96(\mathrm{~m}, 1 \mathrm{H}), 1.08$ $(\mathrm{d}, J=6.9 \mathrm{~Hz}, 3 \mathrm{H}), 1.05(\mathrm{~d}, J=6.0 \mathrm{~Hz}, 3 \mathrm{H}) .{ }^{13} \mathrm{C} \mathrm{NMR} \delta 167.3,133.8,131.9,128.7,127.0,53.5$, 29.9, 19.5, 19.2. Anal. Calcd. For $\mathrm{C}_{12} \mathrm{H}_{16} \mathrm{~N}_{2} \mathrm{O}_{3}$ : C, 61.00; H, 6.83; N, 11.86. Found: C, 61.27; H, $6.90 ; \mathrm{N}, 11.85$.

$\mathrm{N}$-(1-Isopropyl-2-methyl-2-nitropropyl)benzamide (4d). Colorless needles (87\%), mp 125$126{ }^{\circ} \mathrm{C} .{ }^{1} \mathrm{H}$ NMR $\delta 7.87(\mathrm{~d}, J=7.2 \mathrm{~Hz}, 2 \mathrm{H}), 7.59-7.48(\mathrm{~m}, 3 \mathrm{H}), 7.00(\mathrm{~d}, J=10.2 \mathrm{~Hz}, 1 \mathrm{H}), 4.45$ $(\mathrm{dd}, J=10.5,3.0 \mathrm{~Hz}, 1 \mathrm{H}), 2.42-2.17(\mathrm{~m}, 1 \mathrm{H}), 1.74(\mathrm{~s}, 3 \mathrm{H}), 1.65(\mathrm{~s}, 3 \mathrm{H}), 1.03$ (d, $J=6.9 \mathrm{~Hz}$, $3 \mathrm{H}), 0.80(\mathrm{~d}, J=6.6 \mathrm{~Hz}, 3 \mathrm{H}) .{ }^{13} \mathrm{C} \mathrm{NMR} \delta 167.6,133.9,131.9,128.8,126.9,89.6,58.9,28.3$, 26.4, 24.0, 22.9, 15.7. Anal. Calcd. For $\mathrm{C}_{14} \mathrm{H}_{20} \mathrm{~N}_{2} \mathrm{O}_{3}$ : C, 63.62; H, 7.63; N, 10.60. Found: C, $63.91 ; \mathrm{H}, 7.78 ; \mathrm{N}, 10.79$.

$\mathrm{N}$-(1-Isopropyl-2-nitrobutyl)benzamide (4e). Colorless prisms $(96 \%), \mathrm{mp} 159-160{ }^{\circ} \mathrm{C} .{ }^{1} \mathrm{H}$ NMR $\delta 7.76(\mathrm{~d}, J=7.2 \mathrm{~Hz}, 2 \mathrm{H}), 7.53(\mathrm{t}, J=7.5 \mathrm{~Hz}, 1 \mathrm{H}), 7.44(\mathrm{t}, J=7.5 \mathrm{~Hz}, 2 \mathrm{H}), 6.21(\mathrm{~d}, J=$ $9.6 \mathrm{~Hz}, 1 \mathrm{H}), 4.67(\mathrm{dt}, J=8.4,4.5 \mathrm{~Hz}, 1 \mathrm{H}), 4.59-4.51(\mathrm{~m}, 1 \mathrm{H}), 2.22-2.06(\mathrm{~m}, 1 \mathrm{H}), 1.95-1.79$ $(\mathrm{m}, 2 \mathrm{H}), 1.00(\mathrm{~d}, J=6.9 \mathrm{~Hz}, 6 \mathrm{H}), 0.95(\mathrm{t}, J=7.5 \mathrm{~Hz}, 3 \mathrm{H}) .{ }^{13} \mathrm{C}$ NMR $\delta 167.7,133.9,131.9$, 128.7, 126.9, 91.2, 55.6, 28.9, 23.7, 20.2, 16.3, 10.4. Anal. Calcd. For $\mathrm{C}_{14} \mathrm{H}_{20} \mathrm{~N}_{2} \mathrm{O}_{3}$ : C, 63.62; $\mathrm{H}$, 7.63; N, 10.60. Found: C, 63.96; H, 7.91; N, 10.62 .

$\mathrm{N}$-(2-Nitroethyl)benzamide (4f). Colorless plates (54\%), mp 84-85 ${ }^{\circ} \mathrm{C} .{ }^{1} \mathrm{H}$ NMR $\delta 7.76(\mathrm{~d}, J=$ $7.5 \mathrm{~Hz}, 2 \mathrm{H}), 7.55-7.40$ (m, 3H), 6.93 (br s, 1H), 4.66-4.63 (m, 2H), 4.13-3.98 (m, 2H). ${ }^{13} \mathrm{C}$ NMR $\delta$ 168.0, 133.3, 132.0, 128.6, 127.0, 74.5, 37.1. Anal. Calcd. For $\mathrm{C}_{9} \mathrm{H}_{10} \mathrm{~N}_{2} \mathrm{O}_{3}$ : C, 55.67; $\mathrm{H}$, 5.19; N, 14.43. Found: C, 55.96; H, 5.12; N, 14.51 .

$\mathrm{N}$-(2-Methyl-2-nitropropyl)benzamide (4g). Colorless prisms $(86 \%), \mathrm{mp} 121-123{ }^{\circ} \mathrm{C} .{ }^{1} \mathrm{H}$ NMR $\delta 7.76(\mathrm{~d}, J=7.1 \mathrm{~Hz}, 2 \mathrm{H}), 7.54-7.40(\mathrm{~m}, 3 \mathrm{H}), 6.79$ (br s, 1H), 3.95 (d, $J=6.6 \mathrm{~Hz}, 2 \mathrm{H})$, $1.64(\mathrm{~s}, 6 \mathrm{H}) .{ }^{13} \mathrm{C}$ NMR $\delta 167.8,133.6,131.9,128.6,126.9,88.9,46.4,24.0$. Anal. Calcd. For $\mathrm{C}_{11} \mathrm{H}_{14} \mathrm{~N}_{2} \mathrm{O}_{3}$ : C, 59.45; H, 6.35; N, 12.60. Found: C, 59.60; H, 6.42; N, 12.68 .

$\mathrm{N}$-[2-Methyl-1-(1-nitrocyclohexyl)propyl]benzamide (4h). Colorless plates (93\%), $\mathrm{mp}$ 115-116 ${ }^{\circ} \mathrm{C} .{ }^{1} \mathrm{H}$ NMR $\delta 7.87(\mathrm{~d}, \mathrm{~J}=7.8 \mathrm{~Hz}, 2 \mathrm{H}), 7.56-7.47(\mathrm{~m}, 3 \mathrm{H}), 6.79(\mathrm{~d}, J=10.2 \mathrm{~Hz}, 1 \mathrm{H})$, $4.46(\mathrm{~d}, J=10.5 \mathrm{~Hz}, 1 \mathrm{H}), 2.65(\mathrm{~d}, J=12.6 \mathrm{~Hz}, 1 \mathrm{H}), 2.43(\mathrm{~d}, J=14.7 \mathrm{~Hz}, 1 \mathrm{H}), 2.30-2.21(\mathrm{~m}$, $1 \mathrm{H}), 1.86-1.59(\mathrm{~m}, 6 \mathrm{H}), 1.36-1.11(\mathrm{~m}, 2 \mathrm{H}), 1.02(\mathrm{~d}, J=6.6 \mathrm{~Hz}, 3 \mathrm{H}), 0.75(\mathrm{~d}, J=6.6 \mathrm{~Hz}, 3 \mathrm{H})$. ${ }^{13} \mathrm{C}$ NMR $\delta 167.5,133.9,131.9,128.8,126.9,92.3,58.3,34.2,31.8,27.1,24.5,23.4,22.0,21.8$, 15.1. Anal. Calcd. For $\mathrm{C}_{17} \mathrm{H}_{24} \mathrm{~N}_{2} \mathrm{O}_{3}$ : C, 67.08; H, 7.95; N, 9.20. Found: C, 67.27; H, 8.13; N, 9.38 .

Decanoic acid (2-nitro-1-p-tolylpropyl)amide (4i) [two stereoisomers]. Colorless prisms (89\%), mp 79-81 ${ }^{\circ} \mathrm{C} .{ }^{1} \mathrm{H}$ NMR $\delta 7.14-7.09(\mathrm{~m}, 8 \mathrm{H}), 6.63(\mathrm{~d}, J=6.6 \mathrm{~Hz}, 1 \mathrm{H}), 6.45(\mathrm{~d}, J=8.1$ $\mathrm{Hz}, 1 \mathrm{H}), 5.47-5.39(\mathrm{~m}, 2 \mathrm{H}), 5.02-4.91(\mathrm{~m}, 2 \mathrm{H}), 2.32(\mathrm{~s}, 6 \mathrm{H}), 2.26-2.19(\mathrm{~m}, 4 \mathrm{H}), 1.62(\mathrm{t}, J=6.6$ $\mathrm{Hz}, 4 \mathrm{H}), 1.52$ (dd, $J=9.6,6.9 \mathrm{~Hz}, 6 \mathrm{H}), 1.25$ (br s, $24 \mathrm{H}), 0.87$ (t, $J=6.6 \mathrm{~Hz}, 6 \mathrm{H}) .{ }^{13} \mathrm{C} \mathrm{NMR} \delta$ $172.9,172.7,138.7,138.3,134.1,132.7,129.7,129.6,127.0,126.4,86.5,85.3,55.6,54.9,36.7$, 
31.8, 29.4, 29.3, 29.2, 25.7, 25.6, 22.6, 21.1, 17.2, 15.6, 14.1. Anal. Calcd. For $\mathrm{C}_{20} \mathrm{H}_{32} \mathrm{~N}_{2} \mathrm{O}_{3}$ : C, 68.93; H, 9.26; N, 8.04. Found: C, 69.26; H, 9.44; N, 7.99.

$\mathbf{N}$-[3,3-Dimethyl-1-(1-nitroethyl)butyl]benzamide (4j) [two stereoisomers 1:1]. Colorless prisms $(81 \%), \operatorname{mp~} 116-117^{\circ} \mathrm{C} .{ }^{1} \mathrm{H}$ NMR $\delta 7.82-7.76(\mathrm{~m}, 4 \mathrm{H}), 7.57-7.42(\mathrm{~m}, 6 \mathrm{H}), 6.63(\mathrm{~d}, J=$ $9.6 \mathrm{~Hz}, 1 \mathrm{H}), 6.54(\mathrm{~d}, J=8.7 \mathrm{~Hz}, 1 \mathrm{H}), 4.83-4.64(\mathrm{~m}, 3 \mathrm{H}), 4.49$ (td, $J=9.3,3.3 \mathrm{~Hz}, 1 \mathrm{H})$, $1.61-1.51(\mathrm{~m}, 9 \mathrm{H}), 1.35(\mathrm{dd}, J=14.7,9.9 \mathrm{~Hz}, 1 \mathrm{H}), 0.96(\mathrm{~s}, 18 \mathrm{H}) .{ }^{13} \mathrm{C}$ NMR $\delta 167.1,166.8$, $133.9,133.8,131.9,131.9,128.8,128.8,126.9,126.9,87.8,86.3,49.6,48.2,45.9,42.0,30.5$, 30.3, 29.5, 29.5 , 29.5, 16.6, 15.6. Anal. Calcd. For $\mathrm{C}_{15} \mathrm{H}_{22} \mathrm{~N}_{2} \mathrm{O}_{3}$ : C, 64.73; H, 7.97; N, 10.06 . Found: C, 65.04; H, 8.11; N, 10.09 .

$\mathrm{N}$-(2-Nitro-1-thiophen-2-ylbutyl)acetamide (4k) [two stereoisomers]. Colorless microcrystals (58\%), mp 113-115 ${ }^{\circ} \mathrm{C} .{ }^{1} \mathrm{H}$ NMR $\delta ~ 7.28-7.24(\mathrm{~m}, 2 \mathrm{H}), 7.01-6.95(\mathrm{~m}, 4 \mathrm{H}), 6.74(\mathrm{~d}, J=8.5 \mathrm{~Hz}$, $1 \mathrm{H}), 6.36(\mathrm{~d}, J=8.5 \mathrm{~Hz}, 1 \mathrm{H}), 5.84-5.75(\mathrm{~m}, 2 \mathrm{H}), 4.90-4.80(\mathrm{~m}, 2 \mathrm{H}), 2.11-2.03(\mathrm{~m}, 8 \mathrm{H})$, 1.92-1.84 (m, 2H), 1.05-0.97 (m, 6H). ${ }^{13} \mathrm{C}$ NMR $\delta 169.8,169.5,140.3,138.4,127.4,127.2$, 126.5, 125.9, 125.4, 125.2, 92.9, 92.4, 50.7, 50.1, 25.1, 23.9, 23.2 (2C), 10.5, 10.4. Anal. Calcd. For $\mathrm{C}_{10} \mathrm{H}_{14} \mathrm{~N}_{2} \mathrm{O}_{3} \mathrm{~S}: \mathrm{C}, 49.57 ; \mathrm{H}, 5.82 ; \mathrm{N}, 11.56$. Found: $\mathrm{C}, 49.89 ; \mathrm{H}, 5.88 ; \mathrm{N}, 11.28$.

2,2-Dimethyl- $N$-(2-nitro-1-pyridin-3-ylbutyl)propionamide (41). Colorless microcrystals (60\%), mp 192-193 ${ }^{\circ} \mathrm{C} .{ }^{1} \mathrm{H}$ NMR $\delta 8.57(\mathrm{~d}, J=4.8 \mathrm{~Hz}, 2 \mathrm{H}), 7.6(\mathrm{~d}, J=7.4 \mathrm{~Hz}, 1 \mathrm{H}), 7.32-7.27(\mathrm{~m}, 1 \mathrm{H})$, $7.10(\mathrm{~d}, J=9.2 \mathrm{~Hz}, 1 \mathrm{H}), 5.54(\mathrm{dd}, J=4.5 \mathrm{~Hz}, 1 \mathrm{H}), 4.89-4.82(\mathrm{~m}, 1 \mathrm{H}), 2.16-1.82$ (m, 2H), 1.33 $(\mathrm{s}, 9 \mathrm{H}), 1.04(\mathrm{t}, J=7.5 \mathrm{~Hz}, 3 \mathrm{H}) .{ }^{13} \mathrm{C} \mathrm{NMR} \delta 178.2,149.5,147.9,133.8,133.1,123.7,93.0,51.5$, 39.0, 27.4, 25.5, 10.3. Anal. Calcd. For $\mathrm{C}_{14} \mathrm{H}_{21} \mathrm{~N}_{3} \mathrm{O}_{3}$ : C, 60.20; H, 7.58; N, 15.04. Found: C, $60.47 ; \mathrm{H}, 7.80 ; \mathrm{N}, 15.09$.

\section{General procedure for the preparation of $N$ - $(\beta$-cyanoalkyl) amides $6 \mathrm{a}-\mathrm{g}$ and 7}

A mixture of nitrile 5 ( $2 \mathrm{mmol})$ and potassium $t$-butoxide $(0.25 \mathrm{~g}, 2.2 \mathrm{mmol})$ in DMSO $(10 \mathrm{~mL})$ was stirred at room temperature for $1 \mathrm{~h}$. To the resulting solution of $10.645 \mathrm{~g}, 2 \mathrm{mmol}$ ) in DMSO $(10 \mathrm{~mL})$ was added dropwise, and the mixture was stirred at room temperature for $8 \mathrm{hrs}$. The mixture was quenched with water, and extracted with ethyl acetate $(3 \times 30 \mathrm{~mL})$. The extracts were washed with water, dried over $\mathrm{MgSO}_{4}$ and the solvent was removed under reduced pressure. The residue was placed in a silica-gel column and eluted with hexanes/EtOAc 5:1 to give the pure product.

$\mathbf{N}$-[1-(Cyanophenylmethyl)-3,3-dimethylbutyl]benzamide (6a). Colorless plates (78\%), mp 148-150 ${ }^{\circ} \mathrm{C} .{ }^{1} \mathrm{H}$ NMR $\delta 7.84(\mathrm{~d}, J=8.0 \mathrm{~Hz}, 2 \mathrm{H}), 7.56-7.33(\mathrm{~m}, 8 \mathrm{H}), 6.75(\mathrm{~d}, J=7.5 \mathrm{~Hz}, 1 \mathrm{H})$, $4.95(\mathrm{~d}, J=3.6 \mathrm{~Hz}, 1 \mathrm{H}), 4.47-4.40(\mathrm{~m}, 1 \mathrm{H}), 1.69$ (dd, $J=14.4,9.9 \mathrm{~Hz}, 1 \mathrm{H}), 1.49$ (d, $J=14.7$ $\mathrm{Hz}, 1 \mathrm{H}), 0.76$ (s, 9H). ${ }^{13} \mathrm{C}$ NMR $\delta 167.3,133.6,132.4,131.9,129.0,128.6,128.2,127.7,127.0$, 118.9, 51.6, 43.6, 42.1, 30.1, 29.3. Anal. Calcd. For $\mathrm{C}_{21} \mathrm{H}_{24} \mathrm{~N}_{2} \mathrm{O}: \mathrm{C}, 78.71 ; \mathrm{H}, 7.55 ; \mathrm{N}, 8.74$. Found: C, 79.04; H, 7.70; N, 8.85.

$\mathbf{N}$-\{1-[(4-Bromophenyl)cyanomethyl]-3,3-dimethylbutyl\}benzamide (6b). Colorless plates (87\%), mp 155-57 ${ }^{\circ} \mathrm{C} .{ }^{1} \mathrm{H}$ NMR $\delta 7.82-7.79(\mathrm{~m}, 2 \mathrm{H}), 7.58-7.55(\mathrm{~m}, 3 \mathrm{H}), 7.48(\mathrm{t}, J=7.8 \mathrm{~Hz}$, 2H), $7.40(\mathrm{~d}, J=8.4 \mathrm{~Hz}, 2 \mathrm{H}), 6.54(\mathrm{~d}, J=7.5 \mathrm{~Hz}, 1 \mathrm{H}), 4.56(\mathrm{~d}, J=3.9 \mathrm{~Hz}, 1 \mathrm{H}), 4.41-4.34$ (m, $1 \mathrm{H}), 1.66(\mathrm{dd}, J=14.7,10.2 \mathrm{~Hz}, 1 \mathrm{H}), 1.43(\mathrm{~d}, J=14.4 \mathrm{~Hz}, 1 \mathrm{H}), 0.78(\mathrm{~s}, 9 \mathrm{H}) .{ }^{13} \mathrm{C}$ NMR $\delta 167.3$, 
133.4, 132.2, 132.1, 131.5, 129.4, 128.8, 127.0, 118.5, 51.5, 43.2, 42.2, 30.1, 29.4. Anal. Calcd. For $\mathrm{C}_{21} \mathrm{H}_{23} \mathrm{BrN}_{2} \mathrm{O}$ : C, 63.16; H, 5.81; N, 7.02. Found: C, 63.04; H, 5.83; N, 6.90.

$\mathrm{N}$-[1-(Benzo[1,3]dioxol-5-ylcyanomethyl)-3,3-dimethylbutyl]benzamide

(6) [two diastereoisomers isolated]. $1^{\text {st }}$ Diastereoisomer: Colorless prisms $(45 \%), \mathrm{mp} 143-144{ }^{\circ} \mathrm{C} .{ }^{1} \mathrm{H}$ NMR $\delta 7.81-7.77(\mathrm{~m}, 2 \mathrm{H}), 7.58-7.42(\mathrm{~m}, 3 \mathrm{H}), 7.02-6.96(\mathrm{~m}, 2 \mathrm{H}), 6.84(\mathrm{~d}, J=7.8 \mathrm{~Hz}, 1 \mathrm{H}), 6.46$ $(\mathrm{d}, J=7.5 \mathrm{~Hz}, 1 \mathrm{H}), 6.01(\mathrm{~s}, 2 \mathrm{H}), 4.50(\mathrm{~d}, J=3.6 \mathrm{~Hz}, 1 \mathrm{H}), 4.40-4.33(\mathrm{~m}, 1 \mathrm{H}), 1.64-1.49$ (m, 2H), $0.80(\mathrm{~s}, 9 \mathrm{H}) .{ }^{13} \mathrm{C}$ NMR $\delta 167.2,148.2,147.6,133.6,132.1,128.8,126.9,126.0,121.3$, 119.0, 108.6, 108.2, 101.5, 51.6, 43.3, 42.3, 30.2, 29.5. Anal. Calcd. For $\mathrm{C}_{22} \mathrm{H}_{24} \mathrm{~N}_{2} \mathrm{O}_{3}$ : C, 72.50; $\mathrm{H}, 6.64 ; \mathrm{N}, 7.69$. Found: $\mathrm{C}, 72.13 ; \mathrm{H}, 6.64 ; \mathrm{N}, 7.54 .2^{\text {nd }}$ Diastereoisomer: Pale yellow microcrystals (41\%), mp 186-188 ${ }^{\circ} \mathrm{C} .{ }^{1} \mathrm{H}$ NMR $\delta 7.67(\mathrm{~d}, J=7.2 \mathrm{~Hz}, 2 \mathrm{H}), 7.54-7.48(\mathrm{~m}, 1 \mathrm{H})$, 7.45-7.39 (m, 2H), $6.80(\mathrm{~s}, 1 \mathrm{H}), 6.74(\mathrm{~s}, 2 \mathrm{H}), 5.96(\mathrm{~s}, 3 \mathrm{H}), 4.72-4.64(\mathrm{~m}, 1 \mathrm{H}), 4.18(\mathrm{~d}, J=4.5$ $\mathrm{Hz}, 1 \mathrm{H}), 1.78(\mathrm{~d}, J=14.4 \mathrm{~Hz}, 1 \mathrm{H}), 1.42(\mathrm{dd}, J=14.7,9.3 \mathrm{~Hz}, 1 \mathrm{H}), 0.97(\mathrm{~s}, 9 \mathrm{H}) .{ }^{13} \mathrm{C}$ NMR $\delta$ 166.7, 148.1, 147.8, 133.9, 131.8, 128.7, 126.7, 125.4, 122.1, 119.2, 108.4, 103.3, 101.4, 49.8, 45.6, 42.8, 30.5, 29.7. Anal. Calcd. For $\mathrm{C}_{22} \mathrm{H}_{24} \mathrm{~N}_{2} \mathrm{O}_{3}$ : C, 72.50; H, 6.64; N, 7.69. Found: C, 72.37; H, 6.73; N, 7.61.

$\mathrm{N}$-[2-Cyano-1-(2,2-dimethylpropyl)-2-phenylbutyl]benzamide (6d) [two diastereoisomers isolated]. $1^{\text {st }}$ Diastereoisomer: Colorless prisms $(45 \%)$, mp $167-168{ }^{\circ} \mathrm{C} .{ }^{1} \mathrm{H}$ NMR $\delta 7.86(\mathrm{~d}, J=$ $6.9 \mathrm{~Hz}, 2 \mathrm{H}), 7.59-7.33(\mathrm{~m}, 8 \mathrm{H}), 6.41(\mathrm{~d}, J=9.6 \mathrm{~Hz}, 1 \mathrm{H}), 4.80(\mathrm{t}, J=9.6 \mathrm{~Hz}, 1 \mathrm{H}), 2.20-2.12(\mathrm{~m}$, 2H), 1.52 (dd, $J=14.7,10.2 \mathrm{~Hz}, 1 \mathrm{H}), 1.64$ (d, $J=13.8 \mathrm{~Hz}, 1 \mathrm{H}), 0.74$ (t, $J=8.7 \mathrm{~Hz}, 3 \mathrm{H}), 0.73$ (s, 9H). ${ }^{13} \mathrm{C}$ NMR $\delta 167.6,136.3,133.9,131,9,129.0,128.8,128.1,127.0,126.6,120.9,57.1,53.4$, 45.2, 30.5, 30.3, 29.4, 9.6. Anal. Calcd. For $\mathrm{C}_{23} \mathrm{H}_{28} \mathrm{~N}_{2} \mathrm{O}$ : C, 79.27; H, 8.10; N, 8.04. Found: C, 79.38; H, 8.12; N, 8.07. $2^{\text {nd }}$ Diastereoisomer: Colorless microcrystals $(41 \%), m p ~ 153-155{ }^{\circ} \mathrm{C} .{ }^{1} \mathrm{H}$ NMR $\delta 7.55-7.30(\mathrm{~m}, 10 \mathrm{H}), 5.70(\mathrm{~d}, J=9.9 \mathrm{~Hz}, 1 \mathrm{H}), 4.81(\mathrm{t}, J=9.9 \mathrm{~Hz}, 1 \mathrm{H}), 2.31-2.06(\mathrm{~m}$, 2H), 1.96 (d, $J=14.4 \mathrm{~Hz}, 1 \mathrm{H}), 1.16$ (dd, $J=14.4,9.9 \mathrm{~Hz}, 1 \mathrm{H}), 0.95$ (t, $J=2.4 \mathrm{~Hz}, 3 \mathrm{H}), 0.96$ (s, 9H). ${ }^{13} \mathrm{C}$ NMR $\delta 166.5,134.5,134.2,131.5,129.6,128.6,128.2,127.3,126.5,121.4,55.4,52.2$, 46.6, 30.6, 29.6, 29.3, 9.7. Anal. Calcd. For $\mathrm{C}_{23} \mathrm{H}_{28} \mathrm{~N}_{2} \mathrm{O}$ : C, 79.27; H, 8.10; N, 8.04. Found: C, $79.55 ; \mathrm{H}, 8.23 ; \mathrm{N}, 8.06$.

$\mathrm{N}$-(2-Cyano-1-naphthalen-1-yl-2-phenylbutyl)benzamide (6e) [two diastereoisomers isolated]. $1^{\text {st }}$ Diastereoisomer: Colorless prisms $(41 \%), \mathrm{mp} 104-105{ }^{\circ} \mathrm{C} .{ }^{1} \mathrm{H}$ NMR $\delta 8.51(\mathrm{~d}, J=$ $8.4 \mathrm{~Hz}, 1 \mathrm{H}), 7.92-7.83(\mathrm{~m}, 3 \mathrm{H}), 7.73-7.64(\mathrm{~m}, 3 \mathrm{H}), 7.57-7.24(\mathrm{~m}, 10 \mathrm{H}), 6.85(\mathrm{~d}, J=9.0 \mathrm{~Hz}$, $1 \mathrm{H}), 6.76(\mathrm{~d}, J=9.6 \mathrm{~Hz}, 1 \mathrm{H}), 2.09$ (sextet, $J=6.9 \mathrm{~Hz}, 1 \mathrm{H}), 1.59$ (sextet, $J=7.5 \mathrm{~Hz}, 1 \mathrm{H}), 0.91$ (t, $J=7.2 \mathrm{~Hz}, 3 \mathrm{H}) .{ }^{13} \mathrm{C}$ NMR $\delta 166.2,135.9,134.1,133.7,133.6,132.0,131.6,130.7,129.2,129.1$, 129.0, 128.5, 127.2, 127.0, 126.8, 126.0, 125.3, 124.5, 122.8, 120.6, 56.6, 53.2, 30.1, 9.5. Anal. Calcd. For $\mathrm{C}_{28} \mathrm{H}_{24} \mathrm{~N}_{2} \mathrm{O}: \mathrm{N}, 6.93$. Found: $\mathrm{N}, 6.74 .2^{\text {nd }}$ Diastereoisomer: Colorless plates (32\%), mp 214-216 ${ }^{\circ} \mathrm{C} .{ }^{1} \mathrm{H}$ NMR $\delta 8.21(\mathrm{~d}, J=8.21 \mathrm{~Hz}, 1 \mathrm{H}), 7.83(\mathrm{dd}, J=13.2,7.2 \mathrm{~Hz}, 3 \mathrm{H}), 7.68(\mathrm{dd}, J=$ $14.7,8.1 \mathrm{~Hz}, 2 \mathrm{H}), 7.52-7.28(\mathrm{~m}, 8 \mathrm{H}), 7.11-7.01(\mathrm{~m}, 3 \mathrm{H}), 6.90(\mathrm{~d}, J=9.9 \mathrm{~Hz}, 1 \mathrm{H}), 6.93(\mathrm{~d}, J=$ $9.6 \mathrm{~Hz}, 1 \mathrm{H}$ ), 2.53 (sextet, $J=7.5 \mathrm{~Hz}, 1 \mathrm{H}), 2.38$ (sextet, $J=7.2 \mathrm{~Hz}, 1 \mathrm{H}), 0.92$ (t, $J=7.2 \mathrm{~Hz}, 3 \mathrm{H}$ ). ${ }^{13} \mathrm{C}$ NMR $\delta 167.2,135.0,133.7,133.6,133.5,132.0,131.4,128.8,128.7,128.6,127.7,127.1$, 126.7, 126.6, 125.8, 124.9, 124.7, 122.9, 121.9, 55.6, 53.1, 32.8, 9.4. Anal. Calcd. For $\mathrm{C}_{28} \mathrm{H}_{24} \mathrm{~N}_{2} \mathrm{O}$ : C, 83.14; H, 5.98; N, 6.93. Found: C, 82.96; H, 6.02; N, 6.96. 
$\mathbf{N}$-(2-Cyano-2-phenylethyl)benzamide (6f). Colorless plates (58\%), mp 120-122 ${ }^{\circ} \mathrm{C} .{ }^{1} \mathrm{H}$ NMR $\delta 7.78(\mathrm{~d}, J=8.2 \mathrm{~Hz}, 2 \mathrm{H}), 7.53-7.35(\mathrm{~m}, 8 \mathrm{H}), 7.12(\mathrm{t}, J=5.7 \mathrm{~Hz}, 1 \mathrm{H}), 4.37(\mathrm{dd}, J=9.3,6.3 \mathrm{~Hz}$, $1 \mathrm{H}), 3.98-3.90(\mathrm{~m}, 1 \mathrm{H}), 3.67-3.57(\mathrm{~m}, 1 \mathrm{H}) .{ }^{13} \mathrm{C} \mathrm{NMR} \delta 168.1,133.4,132.8,131.9,129.2$, 128.6, 127.5, 127.0, 119.8, 44.7, 37.7. Anal. Calcd. For $\mathrm{C}_{16} \mathrm{H}_{14} \mathrm{~N}_{2} \mathrm{O}$ : C, 76.78; H, 5.64; N, 11.19. Found: C, 76.38; H, 5.69; N, 11.05 .

$\mathrm{N}$-[2-Cyano-2-(2,4-dichloro-phenyl)ethyl]benzamide (6g). Colorless prisms (65\%), mp 136-137 ${ }^{\circ} \mathrm{C} .{ }^{1} \mathrm{H}$ NMR $\delta 7.76(\mathrm{~d}, J=7.3 \mathrm{~Hz}, 2 \mathrm{H}), 7.56-7.41(\mathrm{~m}, 5 \mathrm{H}), 7.31(\mathrm{dd}, J=8.4,2.1 \mathrm{~Hz}$, $1 \mathrm{H}), 6.77(\mathrm{t}, J=6.9 \mathrm{~Hz}, 1 \mathrm{H}), 4.79(\mathrm{dd}, J=8.4,5.7 \mathrm{~Hz}, 1 \mathrm{H}), 4.06-3.97(\mathrm{~m}, 1 \mathrm{H}), 3.76-3.67$ (m, 1H). ${ }^{13} \mathrm{C}$ NMR $\delta 167.9,135.6,134.1,133.3,132.1,130.2(2 \mathrm{C}), 129.2,128.7,128.1,127.0,118.7$, 42.3, 35.2. Anal. Calcd. For $\mathrm{C}_{16} \mathrm{H}_{12} \mathrm{Cl}_{2} \mathrm{~N}_{2} \mathrm{O}$ : C, 60.21; H, 3.69; N, 8.79. Found: C, 60.11; H, 3.70; $\mathrm{N}, 8.50$.

N-(2-Cyano-2-phenylbutyl)benzamide (6h). Colorless prisms (61\%), mp 110-111 ${ }^{\circ} \mathrm{C} .{ }^{1} \mathrm{H}$ NMR $\delta 7.69(\mathrm{~d}, J=7.2 \mathrm{~Hz}, 2 \mathrm{H}), 7.51-7.32(\mathrm{~m}, 8 \mathrm{H}), 6.56(\mathrm{br} \mathrm{s}, 1 \mathrm{H}), 4.21(\mathrm{dd}, J=13.5,7.2 \mathrm{~Hz}$, $1 \mathrm{H}), 3.75(\mathrm{dd}, J=13.5,5.4 \mathrm{~Hz}, 1 \mathrm{H}), 2.21-2.01(\mathrm{~m}, 2 \mathrm{H}), 0.93(\mathrm{t}, J=7.2 \mathrm{~Hz}, 3 \mathrm{H}) .{ }^{13} \mathrm{C}$ NMR $\delta$ 167.6,135.6, 133.7, 131.7, 129.1, 128.5, 128.3, 126.9, 126.2, 121.3, 507, 47.4, 30.2, 9.3. Anal. Calcd. For $\mathrm{C}_{18} \mathrm{H}_{18} \mathrm{~N}_{2} \mathrm{O}$ : C, 77.67; H, 6.52; N, 10.06. Found: C, 77.48; H, 6.62; N, 9.87.

$\mathrm{N}$-[2-Cyano-2-(2,4-dichlorophenyl)-bis-(1-naphthalen-1-yl-ethyl]benzamide (7). Colorless microcrystals (44\%), mp 211-213 ${ }^{\circ} \mathrm{C} .{ }^{1} \mathrm{H}$ NMR $\delta 8.30(\mathrm{~d}, J=8.1 \mathrm{~Hz}, 1 \mathrm{H}), 7.98-7.67(\mathrm{~m}, 9 \mathrm{H})$, $7.59-7.35(\mathrm{~m}, 12 \mathrm{H}), 7.31(\mathrm{~d}, J=1.8 \mathrm{~Hz}, 1 \mathrm{H}), 7.20(\mathrm{dd}, J=8.4,2.1 \mathrm{~Hz}, 1 \mathrm{H}), 6.95-6.80(\mathrm{~m}, 3 \mathrm{H})$, 6.69-6.58 (m, 2H), $5.54(\mathrm{~d}, J=6.9 \mathrm{~Hz}, 1 \mathrm{H}), 5.03(\mathrm{~d}, J=3.9 \mathrm{~Hz}, 1 \mathrm{H}) .{ }^{13} \mathrm{C}$ NMR $\delta 167.2,166.7$, $135.6,135.2$, 134.1, 133.9, 33.8, 133.6, 133.5, 133.4, 133.3, 132.3, 132.1, 132.0, 131.3, 131.1, $130.5,130.0,129.8,129.7,129.5,129.3,129.1,129.7,128.5,127.7,127.3,127.0,126.9,126.4$, 126.2, 125.0, 123.7, 122.6, 121.9, 118.4, 118.3., 49.7, 41.2, 40.0. Anal. Calcd. For $\mathrm{C}_{44} \mathrm{H}_{31} \mathrm{Cl}_{2} \mathrm{~N}_{3} \mathrm{O}_{2}$ : N, 5.96. Found: N, 6.21.

\section{General procedure for the preparation of $N$-acylpropargylamines $11 \mathrm{a}-\mathrm{g}$}

Method A. To a solution of alkyne $9(2 \mathrm{mmol})$ in dry THF $(10 \mathrm{~mL}), n$-BuLi $(2.6 \mathrm{~mL}, 1.6 \mathrm{M}$ in pentane, $4.2 \mathrm{mmol}$ ) was added at $-78{ }^{\circ} \mathrm{C}$. The solution was stirred at $-78{ }^{\circ} \mathrm{C}$ for $1 \mathrm{~h}$, and a solution of $1(2 \mathrm{mmol})$ in THF $(10 \mathrm{~mL})$ was added. The reaction mixture was stirred for $10 \mathrm{~h}$ while the temperature was allowed to rise to $20{ }^{\circ} \mathrm{C}$. After quenching with water $(20 \mathrm{~mL})$ and extraction with EtOAc $(3 \times 25 \mathrm{~mL})$, the combined organic layers were washed with water, dried over $\mathrm{MgSO}_{4}$ and the solvent was removed in vacuo. The resulted oil was subjected to column chromatography (eluent: ethyl acetate/ hexanes $=1: 10$ then 1:5) to give the pure product.

Method B. To a solution of ethylmagnesium bromide ( $5 \mathrm{mmol}$ ) (prepared in situ ) in THF (10 mL), alkyne 9 (2.7 mmol ) was added. The contents were heated under reflux until evolution of ethane was ceased then left to attain room temperature. A solution of $\mathbf{1}(1.5 \mathrm{mmol})$ in THF $(10 \mathrm{~mL}) \mathrm{was}$ added dropwise to Grignard solution and mixture was stirred for $1 \mathrm{~h}$. After quenching with water $(20 \mathrm{~mL})$ and extraction with EtOAc(4x25 mL), the combined organic layers were dried over $\mathrm{MgSO}_{4}$ and solvent removed in vacuo. The resulted oil was subjected to column chromatography ( eluent: hexanes/EtOAc $=5: 1$ ) to give the pure product. 
$\boldsymbol{N}$-(3,3-Dimethyl-1-phenylethynylbutyl)benzamide (11a). Colorless prisms (53\%), mp 118-120 ${ }^{\circ} \mathrm{C} .{ }^{1} \mathrm{H}$ NMR $\delta 7.80(\mathrm{~d}, J=6.9 \mathrm{~Hz}, 2 \mathrm{H}), 7.52-7.40(\mathrm{~m}, 4 \mathrm{H}), 7.31-7.29(\mathrm{~m}, 2 \mathrm{H}), 6.33(\mathrm{~d}$, $J=8.1 \mathrm{~Hz}, 1 \mathrm{H}), 5.30-5.23(\mathrm{~m}, 1 \mathrm{H}), 1.92-1.78(\mathrm{~m}, 2 \mathrm{H}), 1.09(\mathrm{~s}, 9 \mathrm{H}) .{ }^{13} \mathrm{C}$ NMR $\delta 165.9,134.1$, 131.5(2C), 128.4, 128.1(2C), 127.0, 22.7, 90.1, 82.9, 49.9, 39.6, 30.4, 29.7. Anal. Calcd. For $\mathrm{C}_{21} \mathrm{H}_{23} \mathrm{NO}$ : C, 82.58; H, 7.59; N, 4.59. Found: C, 82.48; H, 7.87; N, 4.68.

$\mathrm{N}$-(4-Methyl-2-phenylethynylpentyl)benzamide (11b). Colorless prisms (63\%), mp 95-96 ${ }^{\circ} \mathrm{C}$. ${ }^{1} \mathrm{H}$ NMR $\delta 7.81(\mathrm{~d}, J=7.3 \mathrm{~Hz}, 2 \mathrm{H}), 7.53-7.41(\mathrm{~m}, 5 \mathrm{H}), 7.31-7.27(\mathrm{~m}, 3 \mathrm{H}), 6.36(\mathrm{~d}, J=8.2 \mathrm{~Hz}$, $1 \mathrm{H}), 5.26(\mathrm{q}, J=8.0 \mathrm{~Hz}, 1 \mathrm{H}), 1.94-1.88(\mathrm{~m}, 1 \mathrm{H}), 1.75(\mathrm{t}, J=7.1 \mathrm{~Hz}, 2 \mathrm{H}), 1.02(\mathrm{~d}, J=6.5 \mathrm{~Hz}$, $6 \mathrm{H}) .{ }^{13} \mathrm{C}$ NMR $\delta 166.3,134.2,131.8,131.7,128.6,128.4,128.3,127.0,122.7,88.7,83.2,45.4$, 41.0, 25.4, 23.0, 22.1. Anal. Calcd. For $\mathrm{C}_{20} \mathrm{H}_{21} \mathrm{NO}$ : C, 82.44; H, 7.26; N, 4.81. Found: C, 82.24; H, 7.58; N, 4.89 .

N-(3-Methyl-1-p-tolylethynylbutyl)benzamide (11c). Colorless microcrystals (86\%), mp 112-113 ${ }^{\circ} \mathrm{C} .{ }^{1} \mathrm{H}$ NMR $\delta 7.86(\mathrm{~d}, J=7.0 \mathrm{~Hz}, 2 \mathrm{H}), 7.58-7.62(\mathrm{~m}, 3 \mathrm{H}), 7.37(\mathrm{~d}, J=8.0 \mathrm{~Hz}, 2 \mathrm{H})$, $7.16(\mathrm{~d}, J=8.1 \mathrm{~Hz}, 2 \mathrm{H}), 6.41(\mathrm{~d}, J=8.4 \mathrm{~Hz}, 1 \mathrm{H}), 5.3(\mathrm{q}, J=8.0 \mathrm{~Hz}, 1 \mathrm{H}), 2.39$ (s, 3H), 2.00-1.93 (m, 1H), 1.82-1.75 (m, 2H ), 1.08 (d, $J=1.1 \mathrm{~Hz}, 3 \mathrm{H}), 1.06(\mathrm{~d}, J=1.2 \mathrm{~Hz}, 3 \mathrm{H}) .{ }^{13} \mathrm{C}$ NMR $\delta$ 166.2, 138.4, 134.2, 131.6 (2C), 129.0, 128.5, 127.0, 119.5, 87.9, 83.2, 45.4, 41.0, 25.3, 22.9, 22.0, 21.4. Anal. Calcd. For $\mathrm{C}_{21} \mathrm{H}_{23} \mathrm{NO}$ : C, 82.58; H, 7.59; N, 4.59. Found: C, 82.31; H, $7.80 ; \mathrm{N}, 4.56$

$\boldsymbol{N}$-(1-Isopropyl-3-p-tolylprop-2-ynyl)benzamide (11d). Colorless microcrystals (85\%), mp 128-129 ${ }^{\circ} \mathrm{C} .{ }^{1} \mathrm{H}$ NMR $\delta 7.80(\mathrm{~d}, J=7.0 \mathrm{~Hz}, 2 \mathrm{H}), 7.52-7.43(\mathrm{~m}, 3 \mathrm{H}), 7.33(\mathrm{~d}, J=8.0 \mathrm{~Hz}, 2 \mathrm{H})$, $7.11(\mathrm{~d}, J=8.0 \mathrm{~Hz}, 2 \mathrm{H}) 6.38(\mathrm{~d}, J=8.6 \mathrm{~Hz}, 1 \mathrm{H}), 5.1(\mathrm{dd}, J=8.4,5.4 \mathrm{~Hz}, 1 \mathrm{H}), 2.35(\mathrm{~s}, 3 \mathrm{H})$, $2.20-2.09(\mathrm{~m}, 1 \mathrm{H}), 1.56(\mathrm{~d}, J=6.7 \mathrm{~Hz}, 6 \mathrm{H}) .{ }^{13} \mathrm{C}$ NMR $\delta 166.5,139.4,138.5,131.7,129.1$, 128.7, 127.0, 122.1, 119.58, 86.2, 84.3, 48.3, 33.3, 21.5, 19.1, 17.8. Anal. Calcd. For $\mathrm{C}_{20} \mathrm{H}_{21} \mathrm{NO}$ : C, 82.44; H, 7.26; N, 4.81. Found: C, 82.54; H, 7.49; N, 4.73.

$\mathrm{N}$-(1-Isobutyloct-2-ynyl)benzamide (11e). Colorless oil $(80 \%) .{ }^{1} \mathrm{H}$ NMR $\delta 7.78,(\mathrm{~d}, J=6.0 \mathrm{~Hz}$, 2H), 7.65-7.40 (m, 3H), 6.20 (d, $J=8.5 \mathrm{~Hz}, 1 \mathrm{H}), 4.90$ (q, $J=5.4,3.2 \mathrm{~Hz}, 1 \mathrm{H}), 2.23$ (dt, $J=7.0$, $2.0 \mathrm{~Hz}, 2 \mathrm{H}), 1.86-1.78(\mathrm{~m}, 1 \mathrm{H}), 1.56(\mathrm{t}, J=7.1 \mathrm{~Hz}, 2 \mathrm{H}), 1.58-1.26(\mathrm{~m}, 7 \mathrm{H}), 0.98(\mathrm{~d}, J=6.6 \mathrm{~Hz}$, $6 \mathrm{H}), 0.9(\mathrm{t}, J=7.0 \mathrm{~Hz}, 3 \mathrm{H}) .{ }^{13} \mathrm{C} \mathrm{NMR} \delta 166.1,134.3,131.5,128.5,126.9,83.7,76.4,45.6,40.8$, 31.0, 28.3, 25.3, 22.9, 22.1, 18.6, 14.0. Anal. Calcd. For $\mathrm{C}_{19} \mathrm{H}_{27} \mathrm{NO}$ : C, 79.95; H, 9.53; N, 4.91 . Found: C, C, 80.15; H, 9.96; N, 4.83 .

$\mathrm{N}$-(1-Thiophen-2-yl-non-2-ynyl)acetamide (11f). Colorless microcrystals (41\%), mp 61-62 ${ }^{\circ} \mathrm{C}$. ${ }^{1} \mathrm{H}$ NMR $\delta 7.21(\mathrm{dd}, J=5.0,4.1 \mathrm{~Hz}, 1 \mathrm{H}), 7.13(\mathrm{~d}, J=3.4 \mathrm{~Hz}, 1 \mathrm{H}), 6.93(\mathrm{dd}, J=3.6,1.5 \mathrm{~Hz}, 1 \mathrm{H})$, $6.39(\mathrm{~d}, J=8.5 \mathrm{~Hz}, 1 \mathrm{H}), 2.23(\mathrm{dt}, J=7.0,2.0 \mathrm{~Hz}, 2 \mathrm{H}), 1.99(\mathrm{~s}, 3 \mathrm{H}), 1.56-1.51(\mathrm{~m}, 2 \mathrm{H})$, $1.42-129(\mathrm{~m}, 6 \mathrm{H}), 0.89(\mathrm{t}, J=6.7 \mathrm{~Hz}, 3 \mathrm{H}) .{ }^{13} \mathrm{C}$ NMR $\delta 168.6,143.7126 .6,125.5,125.1,84.9$, 77.4, 40.5, 31.2, 28.4, 28.3, 23.0, 22.4, 18.6, 14.0. Anal. Calcd. For $\mathrm{C}_{15} \mathrm{H}_{21} \mathrm{NOS}$ : C, 68.40; H, 8.04; N, 5.32. Found: C, 68.47; H, 8.20; N, 5.50.

2,2-Dimethyl- $\mathbf{N}$-(3-phenyl-1-pyridin-3-yl-prop-2-ynyl)propionamide (11g). Colorless microcrystals (41\%), mp 151-152 ${ }^{\circ} \mathrm{C} .{ }^{1} \mathrm{H}$ NMR $\delta 8.69(\mathrm{~d}, J=2.0 \mathrm{~Hz}, 1 \mathrm{H}), 8.64(\mathrm{~d}, J=8.2 \mathrm{~Hz}$, $1 \mathrm{H}), 8.52(\mathrm{~d}, J=6.2 \mathrm{~Hz}, 1 \mathrm{H}), 7.88(\mathrm{~d}, J=7.8 \mathrm{~Hz}, 1 \mathrm{H}), 7.50-7.40(\mathrm{~m}, 6 \mathrm{H}), 6.21(\mathrm{~d}, J=8.2 \mathrm{~Hz}$, 1H), $1.14(\mathrm{~s}, 9 \mathrm{H}) .{ }^{13} \mathrm{C}$ NMR $\delta 176.8,148.7,148.1,135.6,134.4,131.5,128.9,128.7,123.6$, 
121.9, 87.9, 83.8, 42.2, 40.3, 38.1, 27.2. Anal. Calcd. For $\mathrm{C}_{19} \mathrm{H}_{20} \mathrm{~N}_{2} \mathrm{O}: \mathrm{C}, 6$ 78.05; $\mathrm{H}, 6.89 ; \mathrm{N}$, 9.58. Found: C, 6 78.14; H, 7.13; N, 9.55.

\section{General procedure for the preparation of esters of $\boldsymbol{\beta}$ - $\boldsymbol{N}$-acylamino acids 13}

Method A. To a solution of ester $12(2 \mathrm{mmol})$ in dry THF $(10 \mathrm{~mL}), n$-BuLi $(2.6 \mathrm{~mL}, 1.6 \mathrm{M}$ in pentane, $4.2 \mathrm{mmol}$ ) was added at $-78{ }^{\circ} \mathrm{C}$. The solution was stirred at $-78{ }^{\circ} \mathrm{C}$ for $1 \mathrm{~h}$, and a solution of $1(0.504 \mathrm{~g}, 2 \mathrm{mmol})$ in THF $(10 \mathrm{~mL})$ was added. The reaction mixture was stirred for $10 \mathrm{~h}$ while the temperature was allowed to rise to $20{ }^{\circ} \mathrm{C}$. After quenching with water $(20 \mathrm{~mL})$ and extraction with EtOAc $(3 \times 25 \mathrm{~mL})$, the combined organic layers were washed with water $(25$ $\mathrm{mL}$ ), dried over $\mathrm{MgSO}_{4}$ and the solvent was removed in vacuo. The resulting oil was subjected to column chromatography (eluent: ethyl acetate/ hexanes $=1: 10$ then 1:5) to give the pure product.

Method B. To a solution of 12 (6 mmol), in dry DMSO $(10 \mathrm{~mL}), t$-BuOK $(0.67 \mathrm{~g}, 6 \mathrm{mmol})$ was added at $25{ }^{\circ} \mathrm{C}$. The solution was stirred at room temperature for $1 \mathrm{~h}$, and a solution of 1 (3 $\mathrm{mmol})$ in DMSO $(10 \mathrm{ml})$ was added. The mixture was stirred for $12 \mathrm{~h}$, quenched with water (20 $\mathrm{mL})$ and extracted with EtOAc $(4 \times 25 \mathrm{~mL})$. The combined organic layers were washed with water, dried over $\mathrm{MgSO}_{4}$ and solvent was removed in vacuo and the resulting oil was subject to column chromatography (eluent: hexanes/ethyacetate $=5: 1$ ) to give the pure product.

3-Benzoylamino-5,5-dimethyl-2-phenylhexanoic acid methyl ester (13a). Colorless prisms (68\%), mp 183-185 ${ }^{\circ} \mathrm{C} .{ }^{1} \mathrm{H}$ NMR $\delta 7.58(\mathrm{~d}, J=6.9 \mathrm{~Hz}, 2 \mathrm{H}), 7.48-7.28(\mathrm{~m}, 8 \mathrm{H}), 6.16(\mathrm{~d}, J=9.2$ $\mathrm{Hz}, 1 \mathrm{H}), 4.70-4.62(\mathrm{~m}, 1 \mathrm{H}), 4.09(\mathrm{~d}, J=6.6 \mathrm{~Hz}, 1 \mathrm{H}), 3.71(\mathrm{~s}, 3 \mathrm{H}), 1.71-1.52(\mathrm{~m}, 2 \mathrm{H}), 0.83(\mathrm{~s}$, 9H). ${ }^{13} \mathrm{C}$ NMR $\delta 172.9,166.6,154.5,136.0,134.9,131.3,129.0,128.5,127.5,126.6,56.9,52.0$, 49.8, 45.2, 30.6, 29.4. Anal. Calcd. For $\mathrm{C}_{22} \mathrm{H}_{27} \mathrm{NO}_{3}$ : C, 74.76; H, 7.70; N, 3.96. Found: C, 74.88; H, 8.01; N, 3.91 .

3-Benzoylamino-5-methyl-2-naphthalen-1-ylhexanoic acid methyl ester (13b). Colorless microcrystals (92\%), mp 161-162 ${ }^{\circ} \mathrm{C} .{ }^{1} \mathrm{H}$ NMR $\delta 8.53(\mathrm{~d}, J=8.5 \mathrm{~Hz}, 1 \mathrm{H}), 7.92(\mathrm{~d}, J=8.4 \mathrm{~Hz}$, $1 \mathrm{H}), 7.85(\mathrm{dd}, J=3.7,2.5 \mathrm{~Hz}, 1 \mathrm{H}), 7.75-7.72(\mathrm{~m}, 1 \mathrm{H}), 7.60-7.39(\mathrm{~m}, 8 \mathrm{H}), 6.40(\mathrm{~d}, J=8.5 \mathrm{~Hz}$, $1 \mathrm{H}), 5.10(\mathrm{~d}, J=6.3 \mathrm{~Hz}, 1 \mathrm{H}), 4.96-4.87(\mathrm{~m}, 1 \mathrm{H}), 3.75(\mathrm{~s}, 3 \mathrm{H}), 2.20-1.95(\mathrm{~m}, 1 \mathrm{H}), 1.66-1.60$ $(\mathrm{m}, 1 \mathrm{H}), 1.45-1.32(\mathrm{~m}, 1 \mathrm{H}), 0.88(\mathrm{~d}, J=6.6 \mathrm{~Hz}, 3 \mathrm{H}), 0.72(\mathrm{~d}, J=6.6 \mathrm{~Hz}, 3 \mathrm{H}) .{ }^{13} \mathrm{C}$ NMR $\delta$ $173.7,167.2$, 134.5, 133.9, 132.2, 131.4, 131.8, 128.9, 128.5, 128.3, 127.0, 126.8, 126.1, 125.9, 125.1, 123.2, 52.0, 51.1, 49.6, 39.9, 25.3, 23.6, 21.1. Anal. Calcd. For $\mathrm{C}_{25} \mathrm{H}_{27} \mathrm{NO}_{3}$ : C, 77.09; $\mathrm{H}$, 6.99; N, 3.60. Found: C, 76.77; H, 6.76; N, 3.57.

3-Benzoylamino-4-methyl-2-naphthalen-1-yl-pentanoic acid methyl ester (13c) (two diastereoisomers 1:3). Colorless microcrystals (89\%), mp 150-152 ${ }^{\circ} \mathrm{C} .{ }^{1} \mathrm{H}$ NMR $\delta 8.23(\mathrm{~d}, J=$ $8.5 \mathrm{~Hz}, 1 \mathrm{H}), 8.07$ (d, $J=8.6 \mathrm{~Hz}, 1 \mathrm{H}), 7.85$ (dd, $J=8.1,8.0 \mathrm{~Hz} 3 \mathrm{H}), 7.79$ (d, $J=7.3 \mathrm{~Hz}, 2 \mathrm{H})$, $6.97(\mathrm{~d}, J=7.5 \mathrm{~Hz}, 3 \mathrm{H}), 5.59$ (d, $J=10.6 \mathrm{~Hz}, 1 \mathrm{H}), 5.21(\mathrm{~d} \mathrm{t}, J=10.6,3.2 \mathrm{~Hz}, 1 \mathrm{H}), 5.00(\mathrm{~d}, J=$ $3.6 \mathrm{~Hz}, 1 \mathrm{H}), 4.78(\mathrm{~d}, J=10.7 \mathrm{~Hz}, 1 \mathrm{H}), 4.08-3.85(\mathrm{~m}, 1 \mathrm{H}), 3.72$ (s, 3H ), 3.69 (s, 3H), 2.15-2.08 $(\mathrm{m}, 2 \mathrm{H}), 1.23(\mathrm{~d}, J=6.73 \mathrm{~Hz}, 1 \mathrm{H}), 1.1(\mathrm{~d}, J=6.9 \mathrm{~Hz}, 4 \mathrm{H}), 1.08(\mathrm{~d}, J=2.9 \mathrm{~Hz}, 4 \mathrm{H}), 1.03(\mathrm{~d}, J=$ $6.7 \mathrm{~Hz}, 1 \mathrm{H}) .{ }^{13} \mathrm{C}$ NMR $\delta 174.4,172.6,167.6,166.7,134.8,134.1,133.9,132.0,131.8,131.7$, $131.2,130.9,129.3,128.5,128.2,126.8,126.7,126.3,126.1,125.9,125.7,125.5,125.0,124.9$, 
122.3, 122.1, 57.0, 55.2, 52.3, 51.8, 48.6, 46.5, 32.4, 31.2, 20.7, 20.2, 19.3, 16.1. Anal. Calcd. For $\mathrm{C}_{24} \mathrm{H}_{25} \mathrm{NO}_{3}$ : C, 76.77; H, 6.71; N, 3.73. Found: C, 76.47; H, 7.06; N, 3.81.

3-Acetylamino-2-phenyl-3-thiophen-2-yl-propionic acid methyl ester (13d). Colorless microcrystals (75\%), mp 166-168 ${ }^{\circ} \mathrm{C} .{ }^{1} \mathrm{H}$ NMR $\delta 7.33-7.30(\mathrm{~m}, 5 \mathrm{H}), 7.18-7.12(\mathrm{~m}, 2 \mathrm{H}), 6.90$ $(\mathrm{d}, J=3.6 \mathrm{~Hz}, 2 \mathrm{H}), 5.78(\mathrm{dd}, J=9.5,5.4 \mathrm{~Hz}, 1 \mathrm{H}), 4.2(\mathrm{~d}, J=5.3 \mathrm{~Hz}, 1 \mathrm{H}), 3.68(\mathrm{~s}, 3 \mathrm{H}), 1.95$ (s, $3 \mathrm{H}) .{ }^{13} \mathrm{C}$ NMR $\delta 173,169.2,144.1,134.9,128.7,128.1,128.0,126.8,124.5,124.4,56.0,52.3$, 51.6, 23.2. Anal. Calcd. For $\mathrm{C}_{16} \mathrm{H}_{17} \mathrm{NO}_{3} \mathrm{~S}$ : C, 63.34; H, 5.65; N, 4.62. Found: C, 62.99; H, 5.66; $\mathrm{N}, 4.51$.

2-Benzoyl-3-benzoylamino-5,5-dimethylhexanoic acid ethyl ester (13e). Colorless plates (84\%), mp 131-132 ${ }^{\circ} \mathrm{C} .{ }^{1} \mathrm{H}$ NMR $\delta 8.11-8.08(\mathrm{~m}, 2 \mathrm{H}), 7.79-7.76(\mathrm{~m}, 2 \mathrm{H}), 7.62-7.41$ (m, $6 \mathrm{H}), 7.11(\mathrm{~d}, J=9.0 \mathrm{~Hz}, 1 \mathrm{H}), 4.92(\mathrm{ddt}, J=9.6,3.4,2.1 \mathrm{~Hz}, 1 \mathrm{H}), 4.80(\mathrm{~d}, J=3.6 \mathrm{~Hz}, 1 \mathrm{H})$, 4.34-4.06 (m, 2H), 1.89 (dd, $J=15.0,9.6 \mathrm{~Hz}, 1 \mathrm{H}), 1.52(\mathrm{dd}, J=14.7,1.8 \mathrm{~Hz}, 1 \mathrm{H}), 1.15(\mathrm{t}, J=$ $7.5 \mathrm{~Hz}, 3 \mathrm{H}), 0.87$ (s, 9H). ${ }^{13} \mathrm{C}$ NMR $\delta 195.5,168.8,166.5,136.0,134.4,134.0,131.5,129.0$, 128.6, 128.5, 126.9, 61.6, 58.1, 47.2, 46.0, 30.6, 29.4, 13.9. Anal. Calcd. For $\mathrm{C}_{24} \mathrm{H}_{29} \mathrm{NO}_{4}$ : C, 72.89; H, 7.39; N, 3.54. Found: C, 73.07; H, 7.54; N, 3.77.

2-(Benzoylamino-p-tolylmethyl)-3-oxobutyric acid methyl ester (13f). Colorless prisms (88\%), mp 193-194 ${ }^{\circ} \mathrm{C} .{ }^{1} \mathrm{H}$ NMR $\delta ~ 7.86-7.79 ~(m, 2 H), 7.51-7.40$ (m, 3H), 7.22 (d, J=8.1 Hz, 2H), 7.12 (d, $J=8.1 \mathrm{~Hz}, 2 \mathrm{H}), 5.92(\mathrm{dd}, J=8.9,5.2 \mathrm{~Hz}, 1 \mathrm{H}), 4.21$ (d, $J=5.1 \mathrm{~Hz}, 1 \mathrm{H}), 3.71$ (s, $3 \mathrm{H}), 2.30(\mathrm{~s}, 3 \mathrm{H}), 2.18(\mathrm{~s}, 3 \mathrm{H}) .{ }^{13} \mathrm{C} \mathrm{NMR} \delta 204.5,167.8,166.7,137.5,136.1,134.1,131.6$, 129.4, 128.6, 127.1, 126.2, 62.0, 52.9, 52.6, 31.4, 21.0. Anal. Calcd. For $\mathrm{C}_{20} \mathrm{H}_{21} \mathrm{NO}_{4}$ : C, 70.78; H, 6.24; N, 4.13. Found: C, 71.08; H, 6.31; N, 4.43 .

2-(Benzoylaminonaphthalen-1-ylmethyl)-3-oxobutyric acid benzyl ester (13g) (two diastereoisomers). Colorless microcrystals (96\%), mp $122-123{ }^{\circ} \mathrm{C} .{ }^{1} \mathrm{H}$ NMR $\delta 8.45(\mathrm{~d}, J=8.7$ $\mathrm{Hz}, 1 \mathrm{H}), 8.26-8.16(\mathrm{~m}, 3 \mathrm{H}), 7.91-7.75(\mathrm{~m}, 8 \mathrm{H}), 7.65-7.34(\mathrm{~m}, 14 \mathrm{H}), 7.31-7.25(\mathrm{~m}, 8 \mathrm{H})$, 7.06-7.04 (m, 2H), $6.88(\mathrm{dd}, J=9,3.6 \mathrm{~Hz}, 1 \mathrm{H}), 6.80(\mathrm{dd}, J=8.4,4.2 \mathrm{~Hz}, 1 \mathrm{H}), 5.18(\mathrm{~s}, 2 \mathrm{H}), 5.05$ $(\mathrm{s}, 2 \mathrm{H}), 4.41(\mathrm{~d}, J=6.0 \mathrm{~Hz}, 1 \mathrm{H}), 4.31(\mathrm{~d}, J=3.0 \mathrm{~Hz}, 1 \mathrm{H}), 2.48(\mathrm{~s}, 3 \mathrm{H}), 2.00(\mathrm{~s}, 3 \mathrm{H}) .{ }^{13} \mathrm{C} \mathrm{NMR} \delta$ 204.9, 200.9, 169.4, 167.3, 166.5, 166.4, 134.8, 134.5, 134.4, 134.1, 133.9, 133.6, 131.8, 131.6, $130.1,129.9,129.3,129.2,128.8,128.7,128.6,128.5,128.4,128.3,128.1,127.1,127.0,127.0$, 125.9, 125.2, 123.9, 123.7, 122.4, 121.9, 67.8, 67.3, 63.0, 60.5, 49.4, 48.1, 31.9, 28.8. Anal. Calcd. For $\mathrm{C}_{29} \mathrm{H}_{25} \mathrm{NO}_{4}$ : C, 77.14; H, 5.58; N, 3.10. Found: C, 76.82; H, 5.43; N, 3.07.

\section{References}

1. Zaugg, H. E. Synthesis 1970, 49.

2. (a) Zaugg, H. E. Synthesis 1984, 49. (b) Speckamp, W. N.; Hiemstra, H. Tetrahedron 1985, 41, 4367. (c) Hiemstra, H.; Speckamp, W. N. In Comprehensive Organic Synthesis, Trost, B. M.; Fleming, I., Eds.; Pergamon Press: Oxford, 1991; Vol. 2, pp 1047. (d) Speckamp, W. N.; Moolenaar, M. J. Tetrahedron 2000, 56, 3817. 
3. (a) Marson, C. M. Arkivoc 2001, (i), 1. (b) Gonzalez-Temprano, I.; Sotomayor, N. J; Lete, E. Synlett 2002, 593. (c) Osante, I.; Lete, E.; Sotomayor, N. Tetrahedron Lett. 2004, 45, 1253.

4. (a) Bogolyubov, A. A.; Chernysheva, N. B.; Nesterov, V. V.; Antipin, M. Y.; Semenov, V. V. Arkivoc 2000, (i), 497. (b) Jung, J.-W.; Shin, D.-Y.; Seo, S.-Y.; Kim, S.-H.; Paek, S.-M.; Jung, J.-K.; Suh, Y.-G.; Tetrahedron Lett. 2005, 46, 573.

5. (a) Venkov, A. P.; Likanov, L. K.; Mollov, N. M. Synthesis 1982, 486. (b) Ardeo, A.; Garcia, E.; Arrasate, S.; Lete, E.; Sotomayor, N. Tetrahedron Lett. 2003, 44, 8445. (c) Gonzalez-Temprano, I.; Osante, I.; Lete, E.; Sotomayor, N. J. Org. Chem. 2004, 69, 3875.

6. Katritzky, A. R.; Drewniak, M.; Lue, P.; J. Org. Chem. 1988, 53, 5854.

7. Katritzky, A. R.; Pernak, J.; Fan, W. Q.; Saczewski, F. J. Org. Chem. 1991, 56, 4439.

8. Katritzky, A. R.; Shobana, N.; Harris, P. A. Org. Prep. Proceed. Int. 1992, 121.

9. (a) Katritzky, A. R.; Takahashi, I.; Fan, W. Q.; Pernak, J. Synthesis 1991, 1147. (b) Katritzky, A. R.; Fan, W. Q.; Black, M.; Pernak, J. J. Org. Chem. 1992, 57, 547.

10. Katritzky, A. R.; Pernak, J.; Fan, W. Q. Synthesis 1991, 868.

11. Katritzky, A. R.; Urogdi, L.; Mayence, A. J. Org. Chem. 1990, 55, 2206.

12. Katritzky, A. R.; Drewniak, M. J. Chem. Soc., Perkin Trans 1 1988, 2339.

13. Krawczyk, H.; Wolf, W. M. Sliwinski, M. J. Chem. Soc., Perkin Trans 1 2002, 2794.

14. Trost, B. M.; Yeh, V. S. C.; Ito, H.; Bremeyer, N. Org. Lett. 2002, 4, 2621.

15. Carey, F. A.; Sundberg, R. J. Advanced Organic Chemistry Part B, $4^{\text {th }}$ Edn.; New York: Kluwer Academic/Plenum Pub., 2000; p 100.

16. Anderson, J. C.; Blake, A. J.; Howell, G. P.; Wilson, C. J. Org. Chem. 2005, 70, 549.

17. Ballini, R.; Petrini, M. Tetrahedron 2004, 60, 1017.

18. Hui, X.; Desrivot, J.; Bories, C.; Loiseau, P. M.; Franck, X.; Hocquemiller, R.; Figadere, B. Bioorg. Med. Chem. Lett. 2006, 16, 815.

19. Gissot, A.; N'Gouela, S.; Matt, C.; Wagner, A.; Mioskowski, C. J. Org. Chem. 2004, 69, 8997.

20. Czekelius, C.; Carreira, E. M. Angew. Chem. Int. Ed. 2005, 44, 612.

21. Katritzky, A. R.; Saczewski, F. Gazzetta Chimica Italiana 1990, 120, 375.

22. (a) Duthaler, R. O. Tetrahedron 1994, 50, 1539. (b) Ballini, R.; Petrini, M. Tetrahedron Lett. 1999, 40, 4449.

23. Reddy, M. V. R.; Mehrotra, B.; Vankar, Y. D. Tetrahedron Lett. 1995, 36, 4861.

24. Easton, C. J.; Roselt, P. D.; Tiekink, E. R. T. Tetrahedron 1995, 51, 7809.

25. Hodgson, D. R. W.; Sanderson, J. M. Chem. Soc. Rev. 2004, 33, 422.

26. (a) Saylik, D.; Campi, E. M.; Donohue, A. C.; Jackson, W. R.; Robinson, A. J. Tetrahedron: Asymmetry 2001, 12, 657. (b) Fondekar, K. P. P.; Volk, F.-J.; Khaliq-uz-Zaman, S. M.;

Bisel, P.; Frahm, A. W. Tetrahedron: Asymmetry 2002, 13, 2241.

27. Kita, Y.; Shibata, N.; Kawano, N.; Yoshida, N.; Matsumoto, K.; Takebe, Y. J. Chem. Soc., Perkin Trans. 1 1996, 2321.

28. Wu, J.; Hou, X.-L.; Dai, L.-X. J. Org. Chem. 2000, 65, 1344. 
29. Kobayashi, S.; Shimizu, H.; Yamashita, Y.; Ishitani, H.; Kobayashi, J. J. Am. Chem. Soc. 2002, 124, 13678.

30. Katritzky, A. R.; Abdel-Fattah, A. A. A.; Vakulenko, A. V.; Tao, H. J. Org. Chem. 2005, 70, 9191.

31. Katritzky, A. R.; Abdel-Fattah, A. A. A.; Wang, M. J. Org. Chem. 2003, 68, 4932.

32. Katritzky, A. R.; Abdel-Fattah, A. A. A.; Steel, P. J. Tetrahedron Lett. 2006, 47, 1465

33. Carlier, P. R.; Lo, K. M.; Lo, M. M.-C.; Williams, I. D. J. Org. Chem. 1995, 60, 7511.

34. Cossy, J.; Schmitt, A.; Cinquin, C.; Buisson, D.; Belotti, D. Bioorg. Med. Chem. Lett. 1997, 7, 1699.

35. (a) Yu, P. H.; Davis, B. A.; Boulton, A. A. J. Med. Chem. 1992, 35, 3705. (b) Shirota, F. N.; DeMaster, E. G.; Nagasawa, H. T. J. Med. Chem. 1979, 22, 463.

36. Stütz, A. Angew. Chem. Int. Ed. 1987, 26, 320.

37. Huffman, M. A.; Yasuda, N.; DeCamp, A. E.; Grabowski, E. J. J. J. Org. Chem. 1995, 60, 1590.

38. (a) Leadbeater, N. E.; Torenius, H. M.; Tye, H. Molecular Diversity 2003, 7, 135. (b) Wei, C.; Li, C.-J. J. Am. Chem. Soc. 2003, 125, 9585. (c) Wei, C.; Li, Z.; Li, C-J. Org. Lett. 2003, 5, 4473. (d) Shi, L.; Tu, Y.-Q.; Wang, M.; Zhang, F.-M.; Fan, C.-A. Org. Lett. 2004, 6, 1001 .

39. Koradin, C.; Polborn, K.; Knochel, P. Angew. Chem. Int. Ed. 2002, 41, 2535.

40. Mahrwald, R.; Quint, S. Tetrahedron Lett. 2001, 42, 1655.

41. Fishcher, C.; Carreira, E. M. Org. Lett. 2001, 3, 4319.

42. Katritzky, A. R.; Gallos, J. K.; Yannakopoulou, K. Synthesis 1989, 31.

43. Ahn, J. H.; Joung, M. J.; Yoon, N. M. J. Org. Chem. 1999, 64, 488.

44. Mukerjee, A. K.; Srivastava, R. C. Synthesis 1973, 327.

45. (a) Tang, W; Zhang, X. Org. lett. 2002, 4, 4159. (b) Zhou, Y.-G.; Tang, W.; Wang, W.-B.; Li, W.; Zhang, X. J. Am. Chem. Soc. 2002, 124, 4952. (c) Hsiao, Y.; Rivera, N. R.; Rosner, T.; Krska, S. W.; Njolito, E.; Wang, F.; Sun, Y.; Armstrong, III, J. D.; Grabowski, E. J. J.; Tillyer, R. D.; Spindler, F.; Malan, C. J. Am. Chem. Soc. 2004, 126, 9918.

46. (a) Wenzel, A. G.; Jacobsen, E. N. J. Am. Chem. Soc. 2002, 124, 12964. (b) Akiyama, T.; Itoh, J.; Yokota, K.; Fuchibe, K. Angew. Chem. Int. Ed. 2004, 43, 1566.

47. Kobayashi, S.; Ishitani, H.; Komiyama, S.; Oniciu, D. C.; Katritzky, A. R. Tetrahedron Lett. 1996, 37, 3731.

48. Katritzky, A. R.; Shobana, N.; Harris, P. A. Tetrahedron Lett. 1990, 31, 3999. 